\title{
gem-Dihalocyclopropanes as Building Blocks in Natural-Product Synthesis: Enantioselective Total Syntheses of ent-Erythramine and 3-epi-Erythramine
}

\author{
Pauline C. Stanislawski, Anthony C. Willis, and Martin G. Banwell*[a]
}

\begin{abstract}
Erythramine ((-)-1), the enantiomer of the alkaloid erythramine, was prepared in 15 steps from known compounds. The first of three pivotal bond-forming steps in the synthesis was a Suzuki-Miyaura cross-coupling reaction of the starting materials to give a bis-silyl ether. The second involved silver(I)-induced electrocyclic ring opening of the gem-dichlorocyclopropane formed in the next step and
\end{abstract}

trapping of the ensuing $\pi$-allyl cation by the tethered nitrogen atom to give, following cleavage of the allyloxycarbonyl protecting group, an approximately 5:6 mixture of the chromatographically separable diastereoisomeric

Keywords: alkaloids • cyclopropanes $\cdot$ radical reactions $\cdot$ spirocyclization $\cdot$ total synthesis spirocyclic products. In the third critical bond-forming reaction, the iodide formed from one of the diastereoisomers underwent a radical-addition/ elimination reaction sequence that led to $(-)-\mathbf{1}$ in $89 \%$ yield. The application of the same sequence of transformations to the other diastereoisomer afforded 3-epi-(+)-erythramine (3-epi(+)-1).

\section{Introduction}

The crystalline compound (+)-erythramine $((+)-\mathbf{1})$ is a representative member of the subset of erythrina alkaloids that

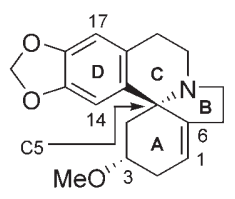

$(+)-1$

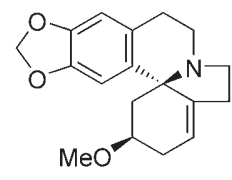

$(-)-1$

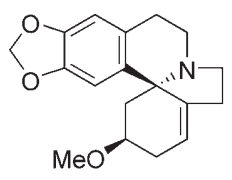

3-epi-(+)-1 contain an aromatic D ring. Its structure was established by using a combination of NMR spectroscopy, mass spectrometry, chemical correlation, and biogenetic studies. ${ }^{[1]}(+)$-Erythramine has been isolated from a variety of plant sources, including the seeds of Erythrina sandwicensis, E. subum-

[a] P. C. Stanislawski, Dr. A. C. Willis, ${ }^{+}$Prof. M. G. Banwell Research School of Chemistry Institute of Advanced Studies The Australian National University Canberra, ACT 0200 (Australia) Fax: $(+61) 2-6125-8114$ E-mail:mgb@rsc.anu.edu.au

$\left.{ }^{+}\right]$Correspondence author for X-ray crystallography: E-mail: willis@rsc.anu.edu.au brans, E. cristagalli, and E. glauca, as well as the pods and trunk bark of E. lithiosperma (Leguminosae). ${ }^{[1,2]}$ Extracts of these and other plants that contain erythrina alkaloids have been used in indigenous medicine, and the alkaloids themselves have been shown to display a range of intriguing biological activities, including curare-like action (attributed to antagonistic effects exerted at neural acetylcholine receptors), hypnotic effects, cardiovascular activity, and molluscicidal properties. ${ }^{[1]}$ Accordingly, a significant amount of effort has been directed towards the development of total syntheses of these natural products and various analogues. ${ }^{[3]}$ Some impressive results have emerged, including those disclosed in very recent publications, ${ }^{[3 c, d]}$ which detail further elegant approaches to the ABCD ring system of these natural products. Despite such activity, a total synthesis of erythramine has yet to be reported. Herein, we describe a synthesis of its nonnatural enantiomer, $(-)-\mathbf{1}$, by a protocol that will allow ready access to a range of alkaloids in the class and in either antipodal form. This study forms part of an ongoing program of our research group to exploit readily available but underutilized gem-dihalocyclopropanes as building blocks for the synthesis of biologically active natural products and their analogues. ${ }^{[4]}$

Our previously reported strategy ${ }^{[3 \mathrm{~b}]}$ for the construction of the $\mathrm{ABCD}$ ring system of the title alkaloids had not been employed hitherto in total synthesis. The pivotal steps associated with our approach to (-)-1 are shown in Scheme 1. In the final stage of the $\mathrm{D} \rightarrow \mathrm{ACD} \rightarrow \mathrm{ABCD}$ ring-forming se- 


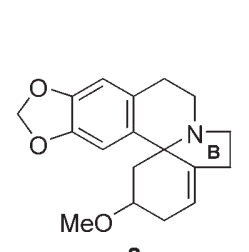

2 5-exo-trig

radical cyclization

then Cl-radical ejection

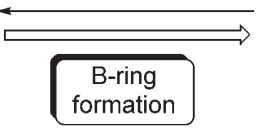

formation

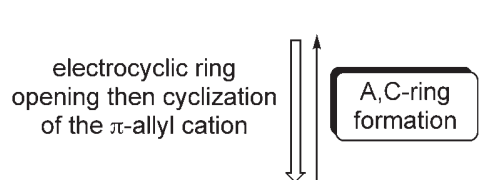

substrate with
intact $D$ ring

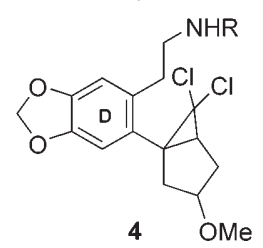

Scheme 1. Retrosynthetic analysis of the erythramine framework 2.

quence, a novel radical-addition/elimination process was expected to install the $\mathrm{B}$ ring with the formation of compound 2 from precursor 3 . The $\Delta^{1(6)}$ alkene required in the target compound (-)-1 would also be incorporated in a regiospecific manner in this radical process. We planned to synthesize the precursor to radical 3 by treating the gem-dichlorocyclopropane 4 with a silver(I) salt. Thus, a $\pi$-allyl cation would be produced through electrocyclic ring opening of the strained three-membered ring and trapped by the pendant nitrogen atom. Such a sequence of events would deliver a spirocyclic product that incorporated the $\mathrm{A}$ and $\mathrm{C}$ rings of the erythrina alkaloids. Of course, a pivotal issue associated with the spirocyclization process is the potential capacity of the methoxy group in substrate $\mathbf{4}$ to exert some level of diastereoselectivity on the reaction and thus establish the required cis relationship between the amine and methoxy groups attached to the A ring.

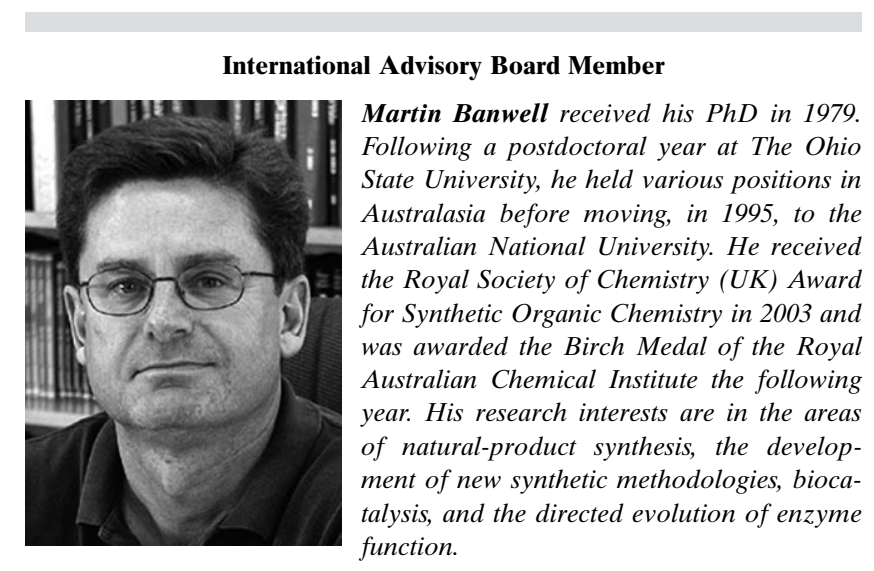

"Asia and Australasia now have a prominent journal, Chemistry-An Asian Journal, as a forum for highlighting the current extraordinary rate of growth of chemical research in the region."

\section{Results and Discussion}

A fundamental consideration in the initial phases of our research was the control of absolute configuration, and in particular whether or not the natural or nonnatural enantiomer of erythramine should be targeted. In the end we chose to prepare the latter compound because the biological properties of (+)-erythramine have been evaluated, whereas those of its enantiomer remain unexplored. Furthermore, if the chiral starting material is available in either enantiomeric form, then any synthesis of (-)-erythramine would also represent a route to the natural product, as proved to be the case.

Suzuki-Miyaura cross-coupling ${ }^{[5]}$ of the reaction partners 5 and $\mathbf{6}$ under standard conditions gave the anticipated product $\mathbf{7}$ in $92 \%$ yield (Scheme 2). A route to substrate $\mathbf{5}$ had been developed during the course of our earlier model studies in this area, ${ }^{[3 b]}$ and compound $\mathbf{6}$ was accessible through chemoenzymatic techniques from cyclopentadiene. ${ }^{[6]}$ The enantiomer of 6 can be prepared through modification of a procedure described by Paquette and co-workers. $^{[7]}$ The TBDMS group of the bis(silyl ether) 7 was removed selectively by using PPTS in ethanol, and the resulting alcohol 8 ( $71 \%)$ was subjected to O-methylation with methyl iodide in the presence of potassium hydride. The required methoxy-substituted cyclopentene 9 was thus obtained in quantitative yield. The treatment of alkene $\mathbf{9}$ with dichlorocarbene generated from chloroform under the phase-transfer conditions described by Makosza and Wawrzyniewicz, ${ }^{[8]}$ with accompanying ultrasonication as recommended by $\mathrm{Xu}$ and Brinker, ${ }^{[9]}$ afforded the anticipated gemdichlorocyclopropane $\mathbf{1 0}(91 \%)$ as an approximately 2:1 mixture of diastereoisomers. As such carbene addition reactions are strongly influenced by steric effects, the isomer with an anti relationship between the methoxy and cyclopropyl groups was presumed to be the major. No effort was made to separate these compounds or their derivatives, because at the relevant point in the synthesis each diastereoisomer was expected to undergo electrocyclic ring opening to give the same $\pi$-allyl cation.

Following the assembly of the requisite carbon framework of the spirocyclization precursor $\mathbf{4}$ (Scheme 1), it was time to introduce the nitrogen-based functionality required as the nucleophile for trapping the cation generated by ring cleavage of the cyclopropyl residue. Our earlier model studies had established that an Alloc-protected primary amine was the most suitable group for this purpose. Accordingly, compound $\mathbf{1 0}$ was treated with tetra- $n$-butylammonium fluoride (TBAF) to provide the corresponding alcohol 11 (97\%) as an approximately 2:1 mixture of diastereoisomers. This mixture was subjected to mesylation under the conditions defined by Crossland and Servis, ${ }^{[10]}$ but with the additional use of DMAP as a catalyst. The resulting esters 12 (100\%) were treated immediately with sodium azide in DMF at $18^{\circ} \mathrm{C}$, and the corresponding mixture of azides $\mathbf{1 3}$ was obtained in $94 \%$ yield. A Staudinger reaction of $\mathbf{1 3}$ with triphenylphosphine in aqueous THF then provided the primary amine $\mathbf{1 4}$, 
<smiles>Oc1cc2c(cc1CCO[SnH3])OCO2</smiles>

5<smiles>C[18O]C1CC=C(O)C1</smiles>

$\frac{\left[\mathrm{PdCl}_{2}(\mathrm{dppf})\right], \mathrm{K}_{3} \mathrm{PO}_{4}}{\mathrm{THF}, 66^{\circ} \mathrm{C}}$

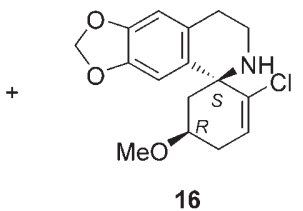

1) LiHMDS, THF, -20 to $0^{\circ} \mathrm{C}, 40 \mathrm{~min}$;

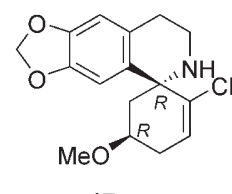

17

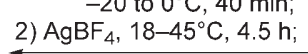

3) $\left[\mathrm{Pd}\left(\mathrm{PPh}_{3}\right)_{4}\right]$, dimedone, THF, $18^{\circ} \mathrm{C}, 16 \mathrm{~h}$
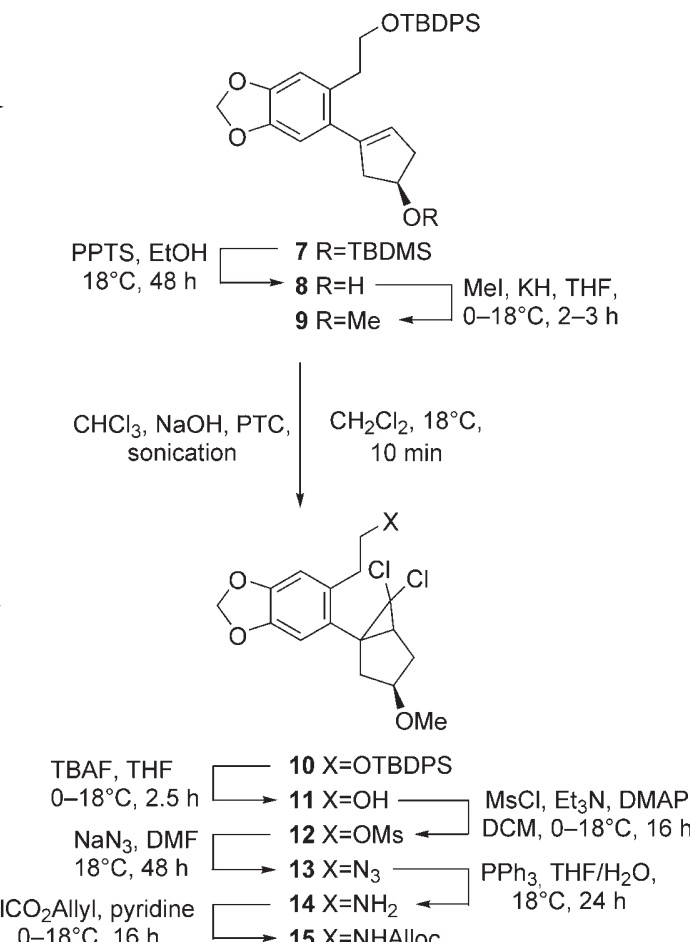

$\mathrm{O}-18^{\circ} \mathrm{C}, 16 \mathrm{~h} \longrightarrow 15 \mathrm{X}=\mathrm{NHAlloc}$

Scheme 2. Synthesis of the spirocyclization substrate $\mathbf{1 5}$ and its conversion into the spirocycles $\mathbf{1 6}$ and $\mathbf{1 7}$. Alloc $=$ allyloxycarbonyl, DMAP $=4-(N, N$-dimethylamino)pyridine, $\mathrm{DMF}=N, N$-dimethylformamide, $\mathrm{dppf}=1,1^{\prime}$-bis(diphenylphosphanyl)ferrocene, LiHMDS $=$ lithium hexamethyldisilazide, Ms $=$ methanesulfonyl, PTC $=$ phase-transfer catalyst, PPTS $=$ pyridinium $p$-toluenesulfonate, TBDPS $=$ tert-butyldiphenylsilyl, TBDMS $=$ tert-butyldimethylsilyl, Tf $=$ trifluoromethanesulfonyl.

which was not purified but, instead, treated immediately with allyl chloroformate in the presence of pyridine to afford the Alloc carbamate $\mathbf{1 5}(\equiv \mathbf{4}$ in which $\mathrm{R}=$ Alloc) in 95\% yield from $\mathbf{1 3}$ and as an approximately 2:1 mixture of diastereoisomers. The ${ }^{13} \mathrm{C}$ NMR $(75 \mathrm{MHz})$ spectrum of compound $\mathbf{1 5}$ revealed twenty signals corresponding to the major diastereoisomer and a series of related resonances attributable to the minor isomer. The most conspicuous feature of the ${ }^{1} \mathrm{H}$ NMR $(300 \mathrm{MHz})$ spectrum was the appearance of two singlets, at $\delta=3.26$ and $3.19 \mathrm{ppm}$, which could be assigned to the hydrogen atoms of the methoxy group. The integration of these two signals indicated a 1:2 ratio of diastereoisomers.

We next investigated the ability of the gem-dichlorocyclopropane 15 to engage in the foreshadowed electrocyclicring-opening/nucleophilic-trapping sequence. On the basis of our earlier model study, ${ }^{[3 b]}$ compound $\mathbf{1 5}$ was first converted into its conjugate base (with LiHMDS), and this latter species was then treated with silver tetrafluoroborate to induce cleavage of the cyclopropane ring. The crude mixture thus obtained was treated with a source of $\mathrm{Pd}^{0}$ and dimedone (conditions defined by Kunz and Unverzagt ${ }^{[11]}$ for the removal of Alloc protecting groups from amines) to give the chromatographically separable diastereoisomeric spirocyclic products $\mathbf{1 6}$ and $\mathbf{1 7}$ in 26 and $30 \%$ yield, respectively. Although the spectroscopic data acquired for these compounds were fully consistent with the assigned structures, they did not enable the unequivocal assignment of configu- ration. Indeed, the configurations of these two compounds were eventually assigned by single-crystal X-ray analysis of a derivative of compound $\mathbf{1 6}$ (see below). The lack of selectivity associated with the transformation of substrate $\mathbf{1 5}$ into amines $\mathbf{1 6}$ and $\mathbf{1 7}$ is disappointing but perhaps unsurprising given the limited steric bulk of the methoxy group and its relative remoteness from the spirocenter that is being assembled.

The reaction sequence used for the elaboration of amine 16, which incorporates the ACD ring system of the erythrina alkaloids, into the target compound (-)-1 is shown in Scheme 3. Once again, the approach used was established in our earlier model studies. ${ }^{[3 b]}$ Thus, amine $\mathbf{1 6}$ was treated with ethylene oxide in methanol to produce the aminoalcohol $\mathbf{1 8}$ in $58 \%$ yield as a crystalline compound suitable for single-crystal X-ray analysis. The ORTEP representation of the crystal structure clearly reveals a cis relationship be-

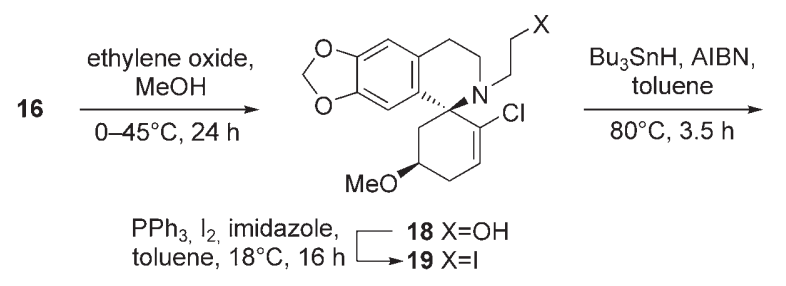

Scheme 3. Completion of the synthesis of ent-erythramine $((-)-\mathbf{1})$. AIBN $=$ azobisisobutyronitrile. 


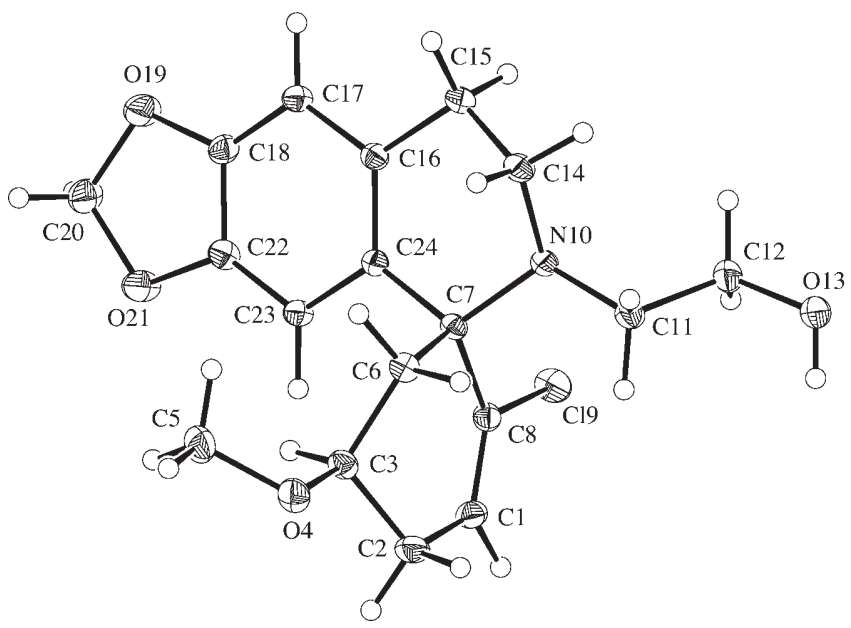

Figure 1. Molecular structure of compound $\mathbf{1 8}$ with selected atoms labeled. Anisotropic displacement ellipsoids are drawn at the $30 \%$ probability level. Hydrogen atoms are drawn as circles with small radii.

tween the methoxy and amine residues on the cyclohexenyl A ring (Figure 1). The alcohol $\mathbf{1 8}$ was converted readily into the corresponding iodide $\mathbf{1 9}$ in $\mathbf{7 5} \%$ yield by using molecular iodine in the presence of imidazole and triphenylphosphine. Compound $\mathbf{1 9}$ was then treated with tri- $n$-butyltin hydride and AIBN in toluene. This final step resulted in the efficient formation of the target compound (-)-1 (89\%) by 5exo-trig radical cyclization/chlorine-radical elimination to ensure the establishment of the associated double bond with complete positional fidelity. The ${ }^{1} \mathrm{H}$ and ${ }^{13} \mathrm{C}$ NMR spectral data obtained for ent-erythramine $((-)-\mathbf{1})$ were in complete accord with the assigned structure and in agreement with the limited amount of analogous data reported for the natural product. The specific rotation of the synthetic material $\left([\alpha]_{\mathrm{D}}^{20}=-187\left(c=1.2 \mathrm{~g}(100 \mathrm{~mL})^{-1}\right.\right.$, EtOH or $\left.\left.\mathrm{CHCl}_{3}\right)\right)$ is comparable in magnitude to that recorded for the natural product $\left([\alpha]_{\mathrm{D}}^{29.5}=+228 \quad\left(c=0.19 \mathrm{~g}(100 \mathrm{~mL})^{-1}\right.\right.$, EtOH $\left.)\right){ }^{[12]}$ The variation between these two values may be attributed, in part, to the difference in the temperature at which the optical rotations were measured. The electron-impact $(70 \mathrm{eV})$ mass spectrum of ent-erythramine $((-)-\mathbf{1})$ showed a molecular ion at $m / z$ 299. The base peak appeared at $m / z, 240$ and almost certainly arises from the successive loss of methyl vinyl ether $(\mathrm{MW}=58)$ and a hydrogen atom. The initial fragmentation most likely involves a retro-Diels-Alder or related process. ${ }^{[13]}$

An identical reaction sequence was used to convert the spirocyclic amine 17 into 3-epi-erythramine (3-epi-(+)-1; Scheme 4). Thus, compound $\mathbf{1 7}$ was treated with ethylene oxide, and the resulting aminoalcohol 20 (79\%) was transformed into the corresponding iodide $\mathbf{2 1}$ (79\%) under the same conditions employed for the conversion of $\mathbf{1 8}$ into $\mathbf{1 9}$. Finally, the treatment of compound $\mathbf{2 1}$ with tri- $n$-butyltin hydride in the presence of AIBN afforded 3-epi-erythramine in quantitative yield. The ${ }^{1} \mathrm{H}$ and ${ }^{13} \mathrm{C}$ NMR spectral data obtained for this previously unreported compound were completely consistent with the assigned structure. The ${ }^{13} \mathrm{C}$ NMR

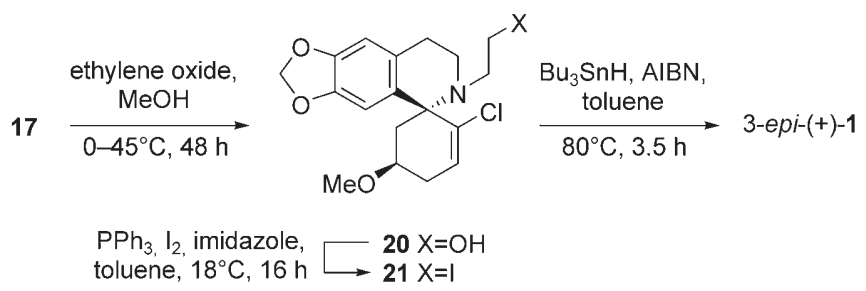

Scheme 4. Completion of the synthesis of 3-epi-erythramine (3-epi-(+)1).

spectrum showed the expected eighteen signals. The key features of the ${ }^{1} \mathrm{H}$ NMR $(800 \mathrm{MHz})$ spectrum are two oneproton singlets at $\delta=6.66$ and $6.48 \mathrm{ppm}$, a one-proton multiplet at $\delta=5.69 \mathrm{ppm}$, and a three-proton singlet at $\delta=$ $3.20 \mathrm{ppm}$. These signals arise from the aromatic hydrogen atoms $14-\mathrm{H}$ and $17-\mathrm{H}$, the olefinic hydrogen atom $1-\mathrm{H}$, and the methoxy group, respectively. As in the case of congener $(-)-\mathbf{1}$, the electron-impact $(70 \mathrm{eV})$ mass spectrum of 3-epierythramine (3-epi-(+)-1) showed a molecular ion at $\mathrm{m} / \mathrm{z}$. 299 , whereas the base peak appeared at $\mathrm{m} / z$ 240. Significantly, the specific rotation of 3-epi-(+)-1 was of large magnitude and positive in sign $\left([\alpha]_{\mathrm{D}}^{20}=+204\left(c=1.0 \mathrm{~g}(100 \mathrm{~mL})^{-1}\right.\right.$, $\left.\mathrm{CHCl}_{3}\right)$ ), as might be expected for a compound that differs from natural erythramine only with respect to the configuration at $\mathrm{C} 3$.

\section{Conclusions}

The silver(I)-induced electrocyclic ring opening of the conjugate base of compound $\mathbf{1 5}$, which incorporates a tethered nitrogen nucleophile, leads to a $\pi$-allyl cation that can be trapped, albeit with little diastereoselectivity, in a spirocyclization process to give a mixture of the Alloc-protected precursors to the amines $\mathbf{1 6}$ and 17. Although the lack of diastereoselectivity detracts from the synthetic approach described herein, the elaboration of these amines to the erythramine analogues (-)-1 and 3-epi-(+)-1 is an attractive means of generating configurationally varied derivatives of the alkaloid that could be used to establish a quantitative-structureactivity-relationship (QSAR) profile for this and related biologically active natural products. Research directed towards this goal is under way in these laboratories.

\section{Experimental Section}

General

Melting points were measured on a Stanford Research Systems Optimelt automated melting-point system and are uncorrected. ${ }^{1} \mathrm{H}$ and ${ }^{13} \mathrm{C}$ NMR spectra were recorded on either a Bruker 800 or a Varian Gemini 300 NMR spectrometer. Unless otherwise specified, spectra were acquired at $20^{\circ} \mathrm{C}$ in deuterochloroform $\left(\mathrm{CDCl}_{3}\right)$ that had been filtered through basic alumina immediately prior to use. Chemical shifts are recorded as $\delta$ values in parts per million $(\mathrm{ppm})$. Infrared spectra were recorded on a Perkin-Elmer 1800 Series FTIR spectrometer, and samples were analyzed as $\mathrm{KBr}$ disks (for solids) or as thin films on $\mathrm{KBr}$ plates (for oils). 
Low-resolution mass spectra were recorded on a Micromass-Waters LCZMD single-quadrupole liquid chromatograph-mass spectrometer or a VG Quattro II triple-quadrupole mass spectrometer by using electronimpact techniques. High-resolution mass spectra were recorded on an Autospec spectrometer. Optical rotations were measured at $20^{\circ} \mathrm{C}$ with a Perkin-Elmer 241 polarimeter at the sodium D line $(589 \mathrm{~nm})$ with the spectroscopic-grade solvents indicated and at the specified concentration (c) defined in $\mathrm{g}(100 \mathrm{~mL})^{-1}$. The measurements were carried out in a cell with a path length of $1 \mathrm{dm}$. Dichloromethane was distilled from calcium hydride, and THF was distilled under nitrogen from sodium benzophenone ketyl. When necessary, reactions were performed under a nitrogen atmosphere.

\section{Syntheses}

6: A magnetically stirred solution of thiourea $(4.03 \mathrm{~g}, 53.0 \mathrm{mmol})$ and rose bengal $(\approx 200 \mathrm{mg})$ in $\mathrm{MeOH}(120 \mathrm{~mL})$ was cooled to $-40^{\circ} \mathrm{C}$ and flushed continuously with oxygen. After $15 \mathrm{~min}$, freshly cracked cyclopentadiene $(5.00 \mathrm{~g}, 75.6 \mathrm{mmol})$ was added, and the resulting mixture was irradiated at $-32^{\circ} \mathrm{C}$ with an IXL $375-\mathrm{W}$ heat lamp for $8 \mathrm{~h}$. Oxygenation was then discontinued, and the reaction mixture was shielded from light and stirred at $18^{\circ} \mathrm{C}$ for $14 \mathrm{~h}$. The solvent was then removed under reduced pressure, and the residue was dissolved in $\mathrm{CH}_{2} \mathrm{Cl}_{2}(70 \mathrm{~mL}) . \mathrm{Et}_{3} \mathrm{~N}$ (42.1 mL, $302 \mathrm{mmol}$ ), DMAP (924 mg, $7.56 \mathrm{mmol})$, and $\mathrm{Ac}_{2} \mathrm{O}(28.5 \mathrm{~mL}$, $302 \mathrm{mmol}$ ) were added sequentially to the resulting solution at $0{ }^{\circ} \mathrm{C}$ under a nitrogen atmosphere with stirring. The reaction mixture was warmed to $18^{\circ} \mathrm{C}$ and stirred for $16 \mathrm{~h}$. $\mathrm{NH}_{4} \mathrm{Cl}(60 \mathrm{~mL}$ of a saturated aqueous solution) was then added, and the mixture was extracted with $\mathrm{CH}_{2} \mathrm{Cl}_{2}$ $(3 \times 100 \mathrm{~mL})$. The combined organic phases were washed with brine $(1 \times$ $50 \mathrm{~mL})$, then dried $\left(\mathrm{Na}_{2} \mathrm{SO}_{4}\right)$, filtered, and concentrated under reduced pressure to give a light-yellow oil. Purification by flash chromatography ( silica, ethyl acetate/hexane $=15: 85 \rightarrow 1: 1)$ gave $\left(1 R^{*}, 3 S^{*}\right)$-4-cyclopentene1,3-diol diacetate ${ }^{[14]}(7.34 \mathrm{~g}, 53 \%$ from cyclopentadiene) as a pale-yellow oil. IR (film): $\tilde{v}=2950,1737,1366,1232,1076,1019 \mathrm{~cm}^{-1} ;{ }^{1} \mathrm{H}$ NMR $\left(300 \mathrm{MHz}, \mathrm{CDCl}_{3}\right): \delta=6.09(\mathrm{~s}, 2 \mathrm{H}), 5.54(\mathrm{~m}, 2 \mathrm{H}), 2.87(\mathrm{dt}, J=15.0$, $7.6 \mathrm{~Hz}, 1 \mathrm{H}), 2.06(\mathrm{~s}, 6 \mathrm{H}), 1.73 \mathrm{ppm}(\mathrm{dt}, J=15.0,3.8 \mathrm{~Hz}, 1 \mathrm{H}) ;{ }^{13} \mathrm{C} \mathrm{NMR}$ $\left(75 \mathrm{MHz}, \mathrm{CDCl}_{3}\right): \delta=170.8(\mathrm{CO}), 134.7(\mathrm{CH}), 76.7(\mathrm{CH}), 37.2\left(\mathrm{CH}_{2}\right)$, $21.2 \mathrm{ppm}\left(\mathrm{CH}_{3}\right)$; MS (EI, $\left.70 \mathrm{eV}\right): m / z(\%): 185(<1)[M+\mathrm{H}]^{+}, 184(<1)$ $[M]^{+}, 169$ (3) $\left[M-\mathrm{CH}_{3}\right]^{+}, 168(20), 153$ (13), 125 (25), 124 (11), 85 (11), $83(30), 82$ (88), 81 (15), 54 (20), 43 (100); HRMS: $m / z$ calcd for $\mathrm{C}_{9} \mathrm{H}_{12} \mathrm{O}_{4}: 184.0736[M]^{+\cdot}$; found: 184.0734 .

Sodium azide $(18 \mathrm{mg}, 277 \mu \mathrm{mol})$ and lyophilized electric-eel acetyl cholinesterase (EEAC; $2.5 \mathrm{mg}, 349$ units $\mathrm{mg}^{-1}$ ) were added sequentially to magnetically stirred sodium dihydrogen phosphate buffer $(187 \mathrm{~mL}$ of a $1.45 \mathrm{M}$ solution) maintained at $18^{\circ} \mathrm{C}$. When the enzyme had dissolved $(\approx 5 \mathrm{~min}),\left(1 R^{*}, 3 S^{*}\right)$-4-cyclopentene-1,3-diol diacetate $(3.74 \mathrm{~g}, 20.3 \mathrm{mmol})$ was added, the reaction flask was sealed, and the mixture was stirred at $18^{\circ} \mathrm{C}$ for $23 \mathrm{~h} . \mathrm{Na}_{2} \mathrm{~S}_{2} \mathrm{O}_{3}(15 \mathrm{~mL}$ of a $10 \%$ aqueous solution) was then added, and the resulting mixture was stirred at $18^{\circ} \mathrm{C}$ for $0.5 \mathrm{~h}$, then extracted with ethyl acetate/diethyl ether $(1: 1,9 \times 100 \mathrm{~mL})$. The combined organic extracts were then dried $\left(\mathrm{Na}_{2} \mathrm{SO}_{4}\right)$, filtered, and concentrated under reduced pressure to give a light-yellow oil. Purification by flash chromatography (silica, ethyl acetate/hexane $=15: 85 \rightarrow 7: 3)$ gave $(1 R, 3 S)$ (+)-4-hydroxycyclopent-2-enyl acetate ${ }^{[14]}(2.41 \mathrm{~g}, 84 \%)$ as a white crystalline solid. $R_{\mathrm{f}}=0.3\left(\mathrm{CH}_{2} \mathrm{Cl}_{2} /\right.$ hexane/ethyl acetate $\left.=2.5: 5.5: 8\right)$; m.p.: 45 $49^{\circ} \mathrm{C}$ (lit. ${ }^{[14 \mathrm{~b}]}$ m.p.: $\left.45-50{ }^{\circ} \mathrm{C}\right) ;[\alpha]_{\mathrm{D}}^{20}=+62.8\left(c=1.0 \mathrm{~g}(100 \mathrm{~mL})^{-1}, \mathrm{CHCl}_{3}\right)$; IR (film): $\tilde{v}=3418$ (br), 2943，1735，1363，1245, 1091，1060, 1019, $981 \mathrm{~cm}^{-1} ;{ }^{1} \mathrm{H}$ NMR $\left(300 \mathrm{MHz}, \mathrm{CDCl}_{3}\right): \delta=6.07(\mathrm{~m}, 1 \mathrm{H}), 5.94(\mathrm{~m}, 1 \mathrm{H})$, $5.46(\mathrm{~m} 1 \mathrm{H}), 4.68(\mathrm{~m}, 1 \mathrm{H}), 2.77(\mathrm{dt}, J=14.4,7.3 \mathrm{~Hz}, 1 \mathrm{H}), 2.42(\mathrm{br} \mathrm{s}, 1 \mathrm{H})$, $2.02(\mathrm{~s}, 3 \mathrm{H}), 1.61 \mathrm{ppm}(\mathrm{dt}, J=14.4,3.9 \mathrm{~Hz}, 1 \mathrm{H}) ;{ }^{13} \mathrm{C} \mathrm{NMR}(75 \mathrm{MHz}$, $\left.\mathrm{CDCl}_{3}\right): \delta=170.9(\mathrm{CO}), 138.6(\mathrm{CH}), 132.4(\mathrm{CH}), 77.2(\mathrm{CH}), 74.7(\mathrm{CH})$, $40.5\left(\mathrm{CH}_{2}\right), 21.2\left(\mathrm{CH}_{3}\right)$; MS (EI, $\left.70 \mathrm{eV}\right): \mathrm{m} / z(\%): 125$ (6) $[\mathrm{M}-\mathrm{HO} \cdot]^{+}, 99$ (10), 83 (30), 82 (100), 81 (22), 55 (20), 54 (17), 53 (21), 43 (57), 39 (10); HRMS: $m / z$ calcd for $\mathrm{C}_{7} \mathrm{H}_{10} \mathrm{O}_{3}: 125.0603\left[M-\mathrm{HO}^{*}\right]^{+}$; found: 125.0604 .

Anhydrous TBDPSCl $(4.52 \mathrm{~mL}, 17.4 \mathrm{mmol})$ was added dropwise to an ice-cooled and magnetically stirred solution of $(1 R, 3 S)-(+)-4$-hydroxycyclopent-2-enyl acetate $(2.24 \mathrm{~g}, 15.8 \mathrm{mmol})$ and imidazole $(4.30 \mathrm{~g}$, $63.2 \mathrm{mmol})$ in $\mathrm{CH}_{2} \mathrm{Cl}_{2}(80 \mathrm{~mL})$ under a nitrogen atmosphere. The reaction mixture was stirred at $0^{\circ} \mathrm{C}$ for approximately $5 \mathrm{~min}$, then warmed to $18^{\circ} \mathrm{C}$ and stirred at this temperature for $1 \mathrm{~h} . \mathrm{NH}_{4} \mathrm{Cl}(100 \mathrm{~mL}$ of a saturated aqueous solution) was then added, and the reaction mixture was extracted with $\mathrm{CH}_{2} \mathrm{Cl}_{2}(2 \times 80 \mathrm{~mL})$. The combined organic phases were dried $\left(\mathrm{Na}_{2} \mathrm{SO}_{4}\right)$, filtered, and concentrated under reduced pressure to give a light-yellow oil, which was purified by flash chromatography ( silica, ethyl acetate/hexane $=0.5: 99.5 \rightarrow 2: 3)$ to give $(1 R, 4 S)-(+)-4-(((1,1-$ dimethylethyl)diphenylsilyl)oxy)-2-cyclopenten-1-ol acetate $\quad(5.88 \mathrm{~g}$, $98 \%$ ) as a clear, colorless oil. $R_{\mathrm{f}}=0.8$ (ethyl acetate/hexane $\left.=2: 3\right) ;[\alpha]_{\mathrm{D}}^{20}=$ +22.0 $\left(c=1.0 \mathrm{~g}(100 \mathrm{~mL})^{-1}, \mathrm{CHCl}_{3}\right)$; IR (film): $\tilde{v}=3071,2932,2892,2858$, 1737, 1428, 1368, 1240, 1110, 1064, 1047, 1021, $702 \mathrm{~cm}^{-1} ;{ }^{1} \mathrm{H}$ NMR $\left(300 \mathrm{MHz}, \mathrm{CDCl}_{3}\right): \delta=7.71-7.67(\mathrm{~m}, 4 \mathrm{H}), 7.45-7.37(\mathrm{~m}, 6 \mathrm{H}), 5.92(\mathrm{~m}$, $1 \mathrm{H}), 5.85(\mathrm{~m}, 1 \mathrm{H}), 5.41-5.38$ (complex m, 1H), 4.71-4.67 (complex m, $1 \mathrm{H}), 2.66(\mathrm{dt}, J=13.7,7.3 \mathrm{~Hz}, 1 \mathrm{H}), 2.07(\mathrm{~s}, 3 \mathrm{H}), 1.75(\mathrm{dt}, J=13.7,4.5 \mathrm{~Hz}$, $1 \mathrm{H}), 1.08 \mathrm{ppm}(\mathrm{s}, 9 \mathrm{H}) ;{ }^{13} \mathrm{C} \mathrm{NMR}\left(75 \mathrm{MHz}, \mathrm{CDCl}_{3}\right): \delta=171.0$ (CO), $138.9(\mathrm{CH}), 135.8(\mathrm{CH}), 134.0\left(\mathrm{C}_{\text {quat }}\right), 131.4(\mathrm{CH}), 129.9(\mathrm{CH}), 127.8$ $(\mathrm{CH}), 76.9(\mathrm{CH}), 75.8(\mathrm{CH}), 41.1\left(\mathrm{CH}_{2}\right), 27.0\left(\mathrm{CH}_{3}\right), 21.3\left(\mathrm{CH}_{3}\right)$, $19.2 \mathrm{ppm}\left(\mathrm{C}_{\text {quat }}\right)$; MS (EI, $\left.70 \mathrm{eV}\right): \mathrm{m} / z(\%): 323(<1)\left[M-\mathrm{C}_{4} \mathrm{H}_{9}\right]^{+}, 263$ (18), 246 (18), 245 (56), 242 (76), 241 (100), 201 (14), 200 (47), 199 (96), 197 (30), 181 (51), 139 (24), 135 (29), 105 (15), 77 (23), 66 (14), 57 (15), 43 (34); HRMS: $m / z$ calcd for $\mathrm{C}_{23} \mathrm{H}_{28} \mathrm{O}_{3} \mathrm{Si}: 323.1103\left[M-\mathrm{C}_{4} \mathrm{H}_{9}\right]^{+}$; found: 323.1097 .

Anhydrous $\mathrm{K}_{2} \mathrm{CO}_{3}(3.21 \mathrm{~g}, 23.2 \mathrm{mmol})$ was added to a magnetically stirred solution of $(1 R, 4 S)-(+)-4-(((1,1-$ dimethylethyl $)$ diphenylsilyl)oxy)2-cyclopenten-1-ol acetate $(5.88 \mathrm{~g}, 15.5 \mathrm{mmol})$ in $\mathrm{MeOH}(50 \mathrm{~mL})$ at $18^{\circ} \mathrm{C}$ under a nitrogen atmosphere. The reaction mixture was stirred for $1 \mathrm{~h}$, then concentrated under reduced pressure, and the residue was partitioned between diethyl ether $(100 \mathrm{~mL})$ and water $(100 \mathrm{~mL})$. The aqueous phase was extracted with diethyl ether $(3 \times 100 \mathrm{~mL})$, and the combined organic extracts were dried $\left(\mathrm{Na}_{2} \mathrm{SO}_{4}\right)$, filtered, and concentrated under reduced pressure. The resulting light-yellow oil was purified by flash chromatography (silica, ethyl acetate/hexane $=7: 93 \rightarrow 3: 7$ ) to afford $(1 R, 4 S)-(-)-4-(((1,1-$ dimethylethyl)diphenylsilyl)oxy)-2-cyclopenten-1-ol $(5.22 \mathrm{~g}, 100 \%)$ as a white crystalline solid. $R_{\mathrm{f}}=0.4$ (ethyl acetate $/ \mathrm{CH}_{2} \mathrm{Cl}_{2} /$ hexane $=2: 2.5: 5.5) ;[\alpha]_{\mathrm{D}}^{20}=-2.0\left(c=1.0 \mathrm{~g}(100 \mathrm{~mL})^{-1}, \mathrm{CHCl}_{3}\right)$; IR (film): $\tilde{v}=3313$ (br), 3070, 2932, 2858, 1427, 1365, 1111, 1069, 1019, 902, $701 \mathrm{~cm}^{-1} ;{ }^{1} \mathrm{H}$ NMR $\left(300 \mathrm{MHz}, \mathrm{CDCl}_{3}\right): \delta=7.73-7.69(\mathrm{~m}, 4 \mathrm{H}), 7.46-7.37$ $(\mathrm{m}, 6 \mathrm{H}), 5.91(\mathrm{~m}, 1 \mathrm{H}), 5.86(\mathrm{~m}, 1 \mathrm{H}), 4.67(\mathrm{~m}, 1 \mathrm{H}), 4.52(\mathrm{~m}, 1 \mathrm{H}), 2.57$ $(\mathrm{dt}, J=13.7,7.1 \mathrm{~Hz}, 1 \mathrm{H}), 1.90(\mathrm{~m}, 1 \mathrm{H}), 1.66(\mathrm{dt}, J=13.7,4.6 \mathrm{~Hz}, 1 \mathrm{H})$, $1.09 \mathrm{ppm}(\mathrm{s}, 9 \mathrm{H}) ;{ }^{13} \mathrm{C} \mathrm{NMR}\left(75 \mathrm{MHz}, \mathrm{CDCl}_{3}\right): \delta=136.9(\mathrm{CH}), 135.9$ $(\mathrm{CH}), 135.7(\mathrm{CH}), 134.0\left(\mathrm{C}_{\text {quat }}\right), 129.8(\mathrm{CH}), 127.8(\mathrm{CH}), 76.1(\mathrm{CH}), 75.1$ $(\mathrm{CH}), 44.6\left(\mathrm{CH}_{2}\right), 27.0\left(\mathrm{CH}_{3}\right), 19.2 \mathrm{ppm}\left(\mathrm{C}_{\text {quat }}\right)$; MS (EI, $\left.70 \mathrm{eV}\right): m / z(\%)$ : $338(<1)[M]^{+\cdot}, 281(87)\left[\mathrm{M}-\mathrm{C}_{4} \mathrm{H}_{9}\right]^{+}, 204(19), 203(74), 201(44), 200$ (94), 199 (100), 197 (39), 183 (11), 181 (41), 143 (14), 141 (19), 139 (84), 135 (26), 121 (20), 105 (20), 78 (14), 77 (50), 66 (33), 57 (31), 45 (19), 41 (11); HRMS: $m / z$ calcd for $\mathrm{C}_{21} \mathrm{H}_{26} \mathrm{O}_{2} \mathrm{Si}: 338.1702[M]^{+\bullet}$; found: 338.1709 .

Dess-Martin periodinane $(216 \mathrm{mg}, 514 \mu \mathrm{mol})$ was added to a magnetically stirred solution of $(1 R, 4 S)-(-)-4-(((1,1-$ dimethylethyl $)$ diphenylsilyl)oxy)-2-cyclopenten-1-ol $(145 \mathrm{mg}, 428 \mu \mathrm{mol})$ in $\mathrm{CH}_{2} \mathrm{Cl}_{2}(8 \mathrm{~mL})$ at $18^{\circ} \mathrm{C}$ under a nitrogen atmosphere. The reaction mixture was stirred for $2 \mathrm{~h}$, then filtered through a pad of celite. The filtrate was concentrated under reduced pressure, and the resulting light-yellow oil was purified by flash chromatography (silica, ethyl acetate/hexane $=1: 99 \rightarrow 2: 23$ ) to give $(4 S)$ (+)-4-(((1,1-dimethylethyl)diphenylsilyl)oxy)-2-cyclopenten-1-one $\mathrm{e}^{[14]}$

$(144 \mathrm{mg}, 96 \%)$ as a clear, colorless oil. $R_{\mathrm{f}}=0.5$ (ethyl acetate $/ \mathrm{CH}_{2} \mathrm{Cl}_{2} /$ hexane $=1: 2.5: 5.5) ;[\alpha]_{\mathrm{D}}^{20}=+5.2\left(c=1.0 \mathrm{~g}(100 \mathrm{~mL})^{-1}, \mathrm{CHCl}_{3}\right) ; \mathrm{IR}($ film $)$ : $\tilde{v}=3071,2932,2858,1723,1472,1428,1355,1182,1108,1070,898$, $702 \mathrm{~cm}^{-1} ;{ }^{1} \mathrm{H}$ NMR $\left(300 \mathrm{MHz}, \mathrm{CDCl}_{3}\right): \delta=7.71-7.66$ (complex m, 4H), 7.45-7.41 (complex m, 6H), $6.12(\mathrm{~m}, 1 \mathrm{H}), 4.95(\mathrm{~m}, 1 \mathrm{H}), 2.56-2.48$ (complex m, 2H), 2.38-2.31 (complex m, 1H), $1.08 \mathrm{ppm}(\mathrm{s}, 9 \mathrm{H}) ;{ }^{13} \mathrm{C} \mathrm{NMR}$ $\left(75 \mathrm{MHz}, \mathrm{CDCl}_{3}\right): \delta=206.5(\mathrm{CO}), 163.7(\mathrm{CH}), 135.8(\mathrm{CH}), 134.6(\mathrm{CH})$, $133.5\left(\mathrm{C}_{\text {quat }}\right), 130.2(\mathrm{CH}), 128.0(\mathrm{CH}), 71.8(\mathrm{CH}), 44.9\left(\mathrm{CH}_{2}\right), 26.9\left(\mathrm{CH}_{3}\right)$, $19.2 \mathrm{ppm}\left(\mathrm{C}_{\text {quat }}\right)$; MS (EI, $\left.70 \mathrm{eV}\right): \mathrm{m} / z(\%): 336(4)[\mathrm{M}]^{+\cdot}, 281$ (38), 280 (73), 279 (100), 261 (30), 249 (31), 223 (45), 201 (58), 199 (74), 197 (41), 183 (26), 174 (52), 173 (84), 167 (58), 157 (27), 141 (55), 135 (20), 105 (30), 81 (40), 77 (49), 53 (50), 45 (24); HRMS: $m / z$ calcd for $\mathrm{C}_{21} \mathrm{H}_{24} \mathrm{O}_{2} \mathrm{Si}$ : $336.1546[M]^{+\cdot}$; found: 336.1542 .

A solution of $(4 S)-(+)-4-(((1,1-$ dimethylethyl)diphenylsilyl)oxy)-2-cyclopenten-1-one $(3.00 \mathrm{~g}, 8.91 \mathrm{mmol})$ and triethylamine $(3.70 \mathrm{~mL}, 26.5 \mathrm{mmol})$ 
in THF $(25 \mathrm{~mL})$ was added dropwise to a stirred solution of L-selectride $(10.7 \mathrm{~mL}$ of a $1.0 \mathrm{M}$ solution in THF, $10.7 \mathrm{mmol})$ in THF $(75 \mathrm{~mL})$ at $-78^{\circ} \mathrm{C}$ under nitrogen, and the resulting solution was stirred at $-78^{\circ} \mathrm{C}$ for $0.5 \mathrm{~h}$. $N$-phenyltrifluoromethanesulfonamide $(3.80 \mathrm{~g}, 10.6 \mathrm{mmol})$ was then added in one portion to the reaction mixture, which was stirred for a further $5 \mathrm{~min}$ at $-78^{\circ} \mathrm{C}$ then allowed warmed to $18^{\circ} \mathrm{C}$ and stirred at $18^{\circ} \mathrm{C}$ for $5 \mathrm{~h} . \mathrm{NaHCO}_{3}(100 \mathrm{~mL}$ of a saturated aqueous solution) was added, the phases were separated, and the aqueous phase was extracted with ethyl acetate $(3 \times 50 \mathrm{~mL})$. The combined organic phases were dried $\left(\mathrm{Na}_{2} \mathrm{SO}_{4}\right)$, filtered, and concentrated under reduced pressure to give a yellow oil, which was purified by flash chromatography (silica, hexane/ ethyl acetate/triethylamine $=96: 3: 1)$ to give $(4 R)-4-(((1,1$-dimethylethyl $)$ diphenylsilyl)oxy)-1-cyclopenten-1-yl trifluoromethanesulfonate $(3.77 \mathrm{~g}$, $90 \%$ ) as a clear, colorless oil. $R_{\mathrm{f}}=0.6$ (ethyl acetate/hexane $\left.=1: 9\right) ;[\alpha]_{\mathrm{D}}^{20}=$ $+13.2\left(c=2.0 \mathrm{~g}(100 \mathrm{~mL})^{-1}, \mathrm{CHCl}_{3}\right) ;$ IR (film): $\tilde{v}=3073,2933,2859,1660$, 1426, 1213, 1142, 1112, 1074, 992, 911, 822, 741, 702, 611, $507 \mathrm{~cm}^{-1}$; ${ }^{1} \mathrm{H}$ NMR $\left(300 \mathrm{MHz}, \mathrm{CDCl}_{3}\right): \delta=7.66(\mathrm{~m}, 4 \mathrm{H}), 7.45-7.41$ (complex m, $6 \mathrm{H}), 5.52$ (br t, $J=2.1 \mathrm{~Hz}, 1 \mathrm{H}), 4.57(\mathrm{~m}, 1 \mathrm{H}), 2.65(\mathrm{~m}, 2 \mathrm{H}), 2.59-2.40$ (complex m, 2H), $1.09 \mathrm{ppm}(\mathrm{s}, 9 \mathrm{H}) ;{ }^{13} \mathrm{CNMR}\left(75 \mathrm{MHz}, \mathrm{CDCl}_{3}\right): \delta=$ $146.5\left(\mathrm{C}_{\text {quat }}\right), 135.8(\mathrm{CH}), 133.8\left(\mathrm{C}_{\text {quat }}\right), 130.0(\mathrm{CH}), 127.9(\mathrm{CH}), 118.7(\mathrm{q}$, $\left.J_{\mathrm{C}, \mathrm{F}}=319 \mathrm{~Hz}, \mathrm{C}_{\text {quat }}\right), 115.4(\mathrm{CH}), 70.6(\mathrm{CH}), 41.1\left(\mathrm{CH}_{2}\right), 38.9\left(\mathrm{CH}_{2}\right), 26.9$ $\left(\mathrm{CH}_{3}\right), 19.2 \mathrm{ppm}\left(\mathrm{C}_{\text {quat }}\right) ; \mathrm{MS}(\mathrm{ESI}+): \mathrm{m} / z(\%): 493(19)[M+\mathrm{Na}]^{+}, 471$ (14) $[M+\mathrm{H}]^{+}, 215(29), 97(20), 60$ (100); elemental analysis: calcd (\%) for $\mathrm{C}_{22} \mathrm{H}_{25} \mathrm{~F}_{3} \mathrm{O}_{4} \mathrm{SSi}$ : C 56.15, H 5.35, F 12.11, S 6.81; found: C $56.18, \mathrm{H}$ 5.40, F 12.02 , S 6.62 .

TBAF (2.12 mL of a $1.0 \mathrm{~m}$ solution in THF, $2.12 \mathrm{mmol})$ was added dropwise to a stirred solution of $(4 R)-4-(((1,1-$ dimethylethyl $)$ diphenylsilyl $)$ oxy)-1-cyclopenten-1-yl trifluoromethanesulfonate $(831 \mathrm{mg}, 1.77 \mathrm{mmol})$ in THF $(10 \mathrm{~mL})$ at $0^{\circ} \mathrm{C}$ under a nitrogen atmosphere. The reaction mixture was stirred at $0^{\circ} \mathrm{C}$ for $5 \mathrm{~min}$, then warmed to $18^{\circ} \mathrm{C}$ and stirred at this temperature for $3 \mathrm{~h}$. $\mathrm{NH}_{4} \mathrm{Cl}$ ( $4 \mathrm{~mL}$ of a saturated aqueous solution) was then added, and the resulting mixture was extracted with ethyl acetate $(3 \times 20 \mathrm{~mL})$. The combined organic phases were washed with brine $(1 \times 20 \mathrm{~mL})$, then dried $\left(\mathrm{Na}_{2} \mathrm{SO}_{4}\right)$, filtered, and concentrated under reduced pressure to give a dark-orange oil. Purification by flash chromatography (silica, ethyl acetate/hexane $=3: 7$ ) gave $(R)$-4-hydroxycyclopenten1-yl trifluoromethanesulfonate $(326 \mathrm{mg}, 79 \%)$ as a pale-yellow liquid. $R_{\mathrm{f}}=0.2 \quad($ ethyl acetate/hexane $=3: 7) ;[\alpha]_{\mathrm{D}}^{20}=+1.8 \quad\left(c=1.0 \mathrm{~g}(100 \mathrm{~mL})^{-1}\right.$, $\mathrm{CHCl}_{3}$ ); IR (film): $\tilde{v}=3352,2938,1662,1423,1213,1140,1118,1052,902$, $838,610 \mathrm{~cm}^{-1}$; ${ }^{1} \mathrm{H}$ NMR $\left(300 \mathrm{MHz}, \mathrm{CDCl}_{3}\right): \delta=5.61(\mathrm{~s}, 1 \mathrm{H}), 4.60$ (br s, $1 \mathrm{H}), 2.98(\mathrm{~m}, 1 \mathrm{H}), 2.90(\mathrm{~m}, 1 \mathrm{H}), 2.54(\mathrm{~d}, J=16.9 \mathrm{~Hz}, 1 \mathrm{H}), 2.38(\mathrm{~d}, J=$ $17.2 \mathrm{~Hz}, 1 \mathrm{H}), 1.80 \mathrm{ppm}$ (br s, $1 \mathrm{H}) ;{ }^{13} \mathrm{C} \mathrm{NMR}\left(75 \mathrm{MHz}, \mathrm{CDCl}_{3}\right): \delta=146.7$ $\left(\mathrm{C}_{\text {quat }}\right), 118.7\left(\mathrm{q}, J_{\mathrm{C}, \mathrm{F}}=319 \mathrm{~Hz}, \mathrm{C}_{\text {quat }}\right), 115.5(\mathrm{CH}), 69.1(\mathrm{CH}), 41.4\left(\mathrm{CH}_{2}\right)$, $38.9 \mathrm{ppm}\left(\mathrm{CH}_{2}\right)$; MS (EI, $\left.70 \mathrm{eV}\right): \mathrm{m} / z(\%): 232$ (5) $[M]^{+*}, 215$ (19), 99 (21), 81 (36), 69 (56), 55 (100), 43 (43); HRMS: $\mathrm{m} / z$ calcd for $\mathrm{C}_{6} \mathrm{H}_{7} \mathrm{~F}_{3} \mathrm{O}_{4} \mathrm{~S}: 232.0017[M]^{+*}$; found: 232.0021 .

Anhydrous TBDMSCl (382 mg, $2.53 \mathrm{mmol})$ was added dropwise to an ice-cooled and magnetically stirred solution of $(R)$-4-hydroxycyclopenten-1-yl trifluoromethanesulfonate $(489 \mathrm{mg}, 2.11 \mathrm{mmol})$ and imidazole ( $316 \mathrm{mg}, 4.64 \mathrm{mmol})$ in $\mathrm{CH}_{2} \mathrm{Cl}_{2}(20 \mathrm{~mL})$ under a nitrogen atmosphere. The reaction mixture was stirred at $0^{\circ} \mathrm{C}$ for approximately $5 \mathrm{~min}$, then warmed to $18^{\circ} \mathrm{C}$ and stirred at this temperature for $1 \mathrm{~h}$. $\mathrm{NH}_{4} \mathrm{Cl}(20 \mathrm{~mL}$ of a saturated aqueous solution) was then added, and the resulting mixture was extracted with $\mathrm{CH}_{2} \mathrm{Cl}_{2}(2 \times 80 \mathrm{~mL})$. The combined organic phases were dried $\left(\mathrm{Na}_{2} \mathrm{SO}_{4}\right)$, filtered, and concentrated under reduced pressure to give a light-yellow oil, which was purified by flash chromatography ( silica, hexane/ethyl acetate/triethylamine $=98: 1: 1)$ to give $(R)-4-(((1,1-$ dimethylethyl)dimethylsilyl)oxy)-1-cyclopenten-1-yl trifluoromethanesulfonate $(6)^{[14]}(652 \mathrm{mg}, 89 \%)$ as a clear, colorless liquid. $R_{\mathrm{f}}=0.3$ (hexane/ ethyl acetate/triethylamine $=98: 1: 1) ;[\alpha]_{\mathrm{D}}^{20}=+2.8 \quad\left(c=1.05 \mathrm{~g}(100 \mathrm{~mL})^{-1}\right.$, $\mathrm{CHCl}_{3}$ ); IR (film): $\tilde{v}=2956,2932,2859,1661,1426,1254,1213,1143$, 1092, 994, 912, 837, 778, $610 \mathrm{~cm}^{-1} ;{ }^{1} \mathrm{H}$ NMR $\left(300 \mathrm{MHz}, \mathrm{CDCl}_{3}\right): \delta=5.55$ (quint, $J=2.1 \mathrm{~Hz}, 1 \mathrm{H}$ ), 4.56 (hept, $J=3.6 \mathrm{~Hz}, 1 \mathrm{H}), 2.90(\mathrm{~m}, 1 \mathrm{H}), 2.69$ $(\mathrm{m}, 1 \mathrm{H}), 2.52(\mathrm{dm}, J=16.4 \mathrm{~Hz}, 1 \mathrm{H}), 2.34(\mathrm{dm}, J=16.5 \mathrm{~Hz}, 1 \mathrm{H}), 0.88(\mathrm{~s}$, $9 \mathrm{H}), 0.06 \mathrm{ppm}(\mathrm{s}, 6 \mathrm{H}) ;{ }^{13} \mathrm{C} \mathrm{NMR}\left(75 \mathrm{MHz}, \mathrm{CDCl}_{3}\right): \delta=146.6\left(\mathrm{C}_{\text {quat }}\right)$, $118.7\left(\mathrm{q}, J_{\mathrm{C}, \mathrm{F}}=345 \mathrm{~Hz}, \mathrm{C}_{\text {quat }}\right), 115.5(\mathrm{CH}), 69.7(\mathrm{CH}), 41.4\left(\mathrm{CH}_{2}\right), 39.2$ $\left(\mathrm{CH}_{2}\right), 25.9\left(\mathrm{CH}_{3}\right), 18.2\left(\mathrm{C}_{\text {quat }}\right),-4.8 \mathrm{ppm}\left(\mathrm{CH}_{3}\right) ; \mathrm{MS}(\mathrm{EI}, 70 \mathrm{eV}): \mathrm{m} / \mathrm{z}$ (\%): $346(36)[M]^{+\cdot}, 345(83)\left[M-\mathrm{H}^{\cdot}\right]^{+}, 309(43), 283(58), 201(50), 197$
(72), 135 (100), 75 (51), 73 (68), 67 (71), 57 (58); HRMS: $m / z$ calcd for $\mathrm{C}_{12} \mathrm{H}_{21} \mathrm{~F}_{3} \mathrm{O}_{4} \mathrm{SSi}: 345.0804\left[M-\mathrm{H}^{\cdot}\right]^{+}$; found: 345.0803 .

7: $\left[\mathrm{PdCl}_{2}(\mathrm{dppf})\right]$ (377 mg, $\left.462 \mu \mathrm{mol}\right), \mathrm{K}_{3} \mathrm{PO}_{4}(980 \mathrm{mg}, 4.62 \mathrm{mmol})$, and 6 (800 mg, $2.31 \mathrm{mmol})$ were added sequentially to a magnetically stirred solution of $5^{[3 \mathrm{~b}]}(2.07 \mathrm{~g}, 4.62 \mathrm{mmol})$ in THF $(22 \mathrm{~mL})$ at room temperature. The resulting mixture was deoxygenated (by using argon in a freezethaw process), heated at reflux under an argon atmosphere for $6 \mathrm{~h}$, then cooled to $18^{\circ} \mathrm{C}$ and partitioned between diethyl ether $(70 \mathrm{~mL})$ and water $(50 \mathrm{~mL})$. The aqueous phase was extracted with diethyl ether $(3 \times$ $100 \mathrm{~mL}$ ), and the combined organic phases were washed with brine $(1 \times$ $80 \mathrm{~mL}$ ), dried $\left(\mathrm{Na}_{2} \mathrm{SO}_{4}\right)$, filtered, and concentrated under reduced pressure to give an orange oil. Purification by flash chromatography (silica, ethyl acetate/hexane $=1: 99 \rightarrow 3: 97)$ gave $\boldsymbol{7}^{1}(1.27 \mathrm{~g}, 92 \%)$ as a pale-yellow oil. $\quad R_{\mathrm{f}}=0.4 \quad($ ethyl acetate/hexane $=5: 95) ; \quad[\alpha]_{\mathrm{D}}^{20}=-11.5 \quad(c=1.0 \mathrm{~g}$ $\left(100 \mathrm{~mL}^{-1}, \mathrm{CHCl}_{3}\right)$; IR (film): $\tilde{v}=2930,2857,1503,1483,1428,1362$, 1251, 1210, 1110, 1044, 938, 835, $702 \mathrm{~cm}^{-1} ;{ }^{1} \mathrm{H}$ NMR (300 MHz, $\mathrm{CDCl}_{3}$ ): $\delta=7.60(\mathrm{~m}, 4 \mathrm{H}), 7.41-7.33($ complex m, 6H) $6.63(\mathrm{~s}, 1 \mathrm{H}), 6.60(\mathrm{~s}, 1 \mathrm{H})$, $5.89(\mathrm{~s}, 2 \mathrm{H}), 5.40(\mathrm{~m}, 1 \mathrm{H}), 4.54(\mathrm{~m}, 1 \mathrm{H}), 3.74(\mathrm{t}, J=7.1 \mathrm{~Hz}, 2 \mathrm{H}), 2.87-$ 2.60 (complex m, 4H), 2.53-2.34 (complex m, 2H), 1.03 (s, 9H), 0.89 (s, $9 \mathrm{H}), 0.07(\mathrm{~s}, 3 \mathrm{H}), 0.06 \mathrm{ppm}(\mathrm{s}, 3 \mathrm{H}) ;{ }^{13} \mathrm{C} \mathrm{NMR}\left(75 \mathrm{MHz}, \mathrm{CDCl}_{3}\right): \delta=$ $146.4\left(\mathrm{C}_{\text {quat }}\right), 145.8\left(\mathrm{C}_{\text {quat }}\right), 140.6\left(\mathrm{C}_{\text {quat }}\right), 135.7(\mathrm{CH}), 133.9\left(\mathrm{C}_{\text {quat }}\right), 132.0$ $\left(\mathrm{C}_{\text {quat }}\right), 130.0\left(\mathrm{C}_{\text {quat }}\right), 129.7(\mathrm{CH}), 127.7(\mathrm{CH}), 126.3(\mathrm{CH}), 110.6(\mathrm{CH})$, 108.6 (CH), $100.9\left(\mathrm{CH}_{2}\right), 72.9(\mathrm{CH}), 65.3\left(\mathrm{CH}_{2}\right), 47.6\left(\mathrm{CH}_{2}\right), 43.3\left(\mathrm{CH}_{2}\right)$, $36.4\left(\mathrm{CH}_{2}\right), 27.0\left(\mathrm{CH}_{3}\right), 26.1\left(\mathrm{CH}_{3}\right), 19.3\left(\mathrm{C}_{\text {quat }}\right), 18.3\left(\mathrm{C}_{\text {quat }}\right),-4.5\left(\mathrm{CH}_{3}\right)$, $-4.6 \mathrm{ppm}\left(\mathrm{CH}_{3}\right)$; MS (EI, $\left.70 \mathrm{eV}\right): \mathrm{m} / z(\%): 600(13)[M]^{+}, 599$ (3), 544 (15), 543 (30), 423 (25), 411 (19), 343 (16), 292 (25), 291 (70), 271 (26), 214 (59), 213 (100), 197 (69), 183 (50), 181 (26), 155 (41), 135 (60), 117 (23), 105 (27); HRMS: $m / z$ calcd for $\mathrm{C}_{36} \mathrm{H}_{48} \mathrm{O}_{4} \mathrm{Si}_{2}: 600.3091[M]^{+\cdot}$; found: 600.3090 .

8: PPTS ( $337 \mathrm{mg}, 1.34 \mathrm{mmol}$ ) was added to a magnetically stirred solution of $7(807 \mathrm{mg}, 1.34 \mathrm{mmol})$ in $\mathrm{EtOH}(9 \mathrm{~mL})$ at $18^{\circ} \mathrm{C}$, and the resulting mixture was stirred at this temperature for $48 \mathrm{~h}$. The solvent was then removed under reduced pressure, and the residue was dissolved in ethyl acetate $(1 \times 50 \mathrm{~mL})$. The resulting solution was washed with brine $(1 \times$ $15 \mathrm{~mL})$, dried $\left(\mathrm{Na}_{2} \mathrm{SO}_{4}\right)$, filtered, and concentrated under reduced pressure to give a yellow oil, which was purified by flash chromatography (silica, ethyl acetate/hexane $=1: 4)$ to give $8(463 \mathrm{mg}, 71 \%$ ) as a paleyellow oil. $R_{\mathrm{f}}=0.2$ (ethyl acetate/hexane $\left.=1: 4\right) ;[\alpha]_{\mathrm{D}}^{20}=-6.9 \quad(c=1.0 \mathrm{~g}$ $\left.(100 \mathrm{~mL})^{-1}, \mathrm{CHCl}_{3}\right)$; IR (film): $\tilde{v}=3339,3070,3048,2930,2858,1502$, 1484, 1427, 1213, 1111, 1086, 1043, 937, 823, 739, $702 \mathrm{~cm}^{-1} ;{ }^{1} \mathrm{H}$ NMR $\left(300 \mathrm{MHz}, \mathrm{CDCl}_{3}\right): \delta=7.62-7.59(\mathrm{~m}, 4 \mathrm{H}), 7.42-7.33(\mathrm{~m}, 6 \mathrm{H}), 6.65(\mathrm{~s}$, $1 \mathrm{H}), 6.62(\mathrm{~s}, 1 \mathrm{H}), 5.90(\mathrm{~s}, 2 \mathrm{H}), 5.46(\mathrm{~m}, 1 \mathrm{H}), 4.51(\mathrm{~m}, 1 \mathrm{H}), 3.75(\mathrm{t}, J=$ $7.2 \mathrm{~Hz}, 2 \mathrm{H}), 2.87-2.71(\mathrm{~m}, 4 \mathrm{H}), 2.47-2.36(\mathrm{~m}, 2 \mathrm{H}), 1.03 \mathrm{ppm}(\mathrm{s}, 9 \mathrm{H})$; no signal was observed for the hydrogen atom of the hydroxy group; ${ }^{13} \mathrm{C}$ NMR $\left(75 \mathrm{MHz}, \mathrm{CDCl}_{3}\right): \delta=146.4\left(\mathrm{C}_{\text {quat }}\right), 145.8\left(\mathrm{C}_{\text {quat }}\right), 140.5\left(\mathrm{C}_{\text {quat }}\right)$, $135.7(\mathrm{CH}), 133.8\left(\mathrm{C}_{\text {quat }}\right), 131.5\left(\mathrm{C}_{\text {quat }}\right), 130.0\left(\mathrm{C}_{\text {quat }}\right), 129.7(\mathrm{CH}), 127.7$ $(\mathrm{CH}), 126.1(\mathrm{CH}), 110.6(\mathrm{CH}), 108.5(\mathrm{CH}), 100.9\left(\mathrm{CH}_{2}\right), 72.0(\mathrm{CH}), 65.2$ $\left(\mathrm{CH}_{2}\right), 47.6\left(\mathrm{CH}_{2}\right), 43.3\left(\mathrm{CH}_{2}\right), 36.5\left(\mathrm{CH}_{2}\right), 27.0\left(\mathrm{CH}_{3}\right), 19.2 \mathrm{ppm}\left(\mathrm{C}_{\text {quat }}\right)$; MS (EI, $70 \mathrm{eV}): m / z(\%): 486(10)[M]^{+*}, 429$ (25), 411 (29), 333 (23), 214 (71), 213 (100), 199 (87), 183 (70), 181 (40), 155 (72), 135 (56), 115 (30), 105 (24), 91 (53), 77 (27), 57 (19); HRMS: $m / z$ calcd for $\mathrm{C}_{30} \mathrm{H}_{34} \mathrm{O}_{4} \mathrm{Si}: 486.2226[M]^{+\cdot}$; found: 486.2223 .

9: A solution of the alcohol $8(1.20 \mathrm{~g}, 2.47 \mathrm{mmol})$ and $\mathrm{MeI}(770 \mu \mathrm{L}$, $12.4 \mathrm{mmol})$ in THF $(15 \mathrm{~mL})$ was added dropwise to an ice-cooled and magnetically stirred suspension of $\mathrm{KH}(\approx 2 \mathrm{~g}, 49.9 \mathrm{mmol})$ in $\mathrm{THF}$ $(32 \mathrm{~mL})$ under a nitrogen atmosphere. The resulting mixture was stirred at $0^{\circ} \mathrm{C}$ for approximately $10 \mathrm{~min}$, then warmed to $18^{\circ} \mathrm{C}$ and stirred at this temperature for $3 \mathrm{~h}$. The reaction mixture was then cooled to $0^{\circ} \mathrm{C}$, and $\mathrm{NH}_{4} \mathrm{Cl}(50 \mathrm{~mL}$ of a saturated aqueous solution) was added dropwise. The resulting mixture was extracted with diethyl ether $(3 \times 60 \mathrm{~mL})$, and the combined organic extracts were dried $\left(\mathrm{Na}_{2} \mathrm{SO}_{4}\right)$, filtered, and concentrated under reduced pressure to give a yellow-orange oil. Purification by flash chromatography (silica, ethyl acetate/hexane=5:95) gave $9(1.24 \mathrm{~g}$, $100 \%$ ) as a clear, colorless oil. $R_{\mathrm{f}}=0.1$ (ethyl acetate/hexane $=5: 95$ ); $[\alpha]_{\mathrm{D}}^{20}=-5.5\left(c=1.0 \mathrm{~g}(100 \mathrm{~mL})^{-1}, \mathrm{CHCl}_{3}\right) ; \mathrm{IR}($ film $): \tilde{v}=3070,3048,2930$, $2894,2858,1502,1484,1427,1360,1211,1111,1043,936,703 \mathrm{~cm}^{-1}$;

This material was contaminated with a small amount $(\leq 5 \%)$ of the compound derived from protiodeborylation of the boronic acid $\mathbf{5}$. 
${ }^{1} \mathrm{H}$ NMR $\left(300 \mathrm{MHz}, \mathrm{CDCl}_{3}\right): \delta=7.60(\mathrm{~m}, 4 \mathrm{H}), 7.44-7.33(\mathrm{~m}, 6 \mathrm{H}), 6.64(\mathrm{~s}$, $1 \mathrm{H}), 6.62(\mathrm{~s}, 1 \mathrm{H}), 5.89(\mathrm{~s}, 2 \mathrm{H}), 5.43(\mathrm{~m}, 1 \mathrm{H}), 4.11$ (hept, $J=3.6 \mathrm{~Hz}, 1 \mathrm{H})$, $3.75(\mathrm{t}, J=7.3 \mathrm{~Hz}, 2 \mathrm{H}), 3.31(\mathrm{~s}, 3 \mathrm{H}), 2.89-2.78$ (complex m, 2H), 2.762.40 (complex m, 4H), $1.03 \mathrm{ppm}(\mathrm{s}, 9 \mathrm{H}) ;{ }^{13} \mathrm{C} \mathrm{NMR}\left(75 \mathrm{MHz}, \mathrm{CDCl}_{3}\right): \delta=$ $146.4\left(\mathrm{C}_{\text {quat }}\right), 145.8\left(\mathrm{C}_{\text {quat }}\right), 140.5\left(\mathrm{C}_{\text {quat }}\right), 135.7(\mathrm{CH}), 133.9\left(\mathrm{C}_{\text {quat }}\right), 131.6$ $\left(\mathrm{C}_{\text {quat }}\right), 130.0\left(\mathrm{C}_{\text {quat }}\right), 129.7(\mathrm{CH}), 127.7(\mathrm{CH}), 126.3(\mathrm{CH}), 110.5(\mathrm{CH})$, $108.5(\mathrm{CH}), 100.9\left(\mathrm{CH}_{2}\right), 80.9(\mathrm{CH}), 65.3\left(\mathrm{CH}_{2}\right), 56.4\left(\mathrm{CH}_{3}\right), 43.8\left(\mathrm{CH}_{2}\right)$, $39.7\left(\mathrm{CH}_{2}\right), 36.5\left(\mathrm{CH}_{2}\right), 27.0\left(\mathrm{CH}_{3}\right), 19.3 \mathrm{ppm}\left(\mathrm{C}_{\text {quat }}\right)$; MS (EI, $\left.70 \mathrm{eV}\right): \mathrm{m} / z$ (\%): 500 (46) $\left[M^{+*}, 444\right.$ (23), 443 (54), 215 (32), 214 (92), 213 (100), 199 (82), 183 (95), 181 (50), 155 (85), 135 (66), 115 (40), 91 (52), 77 (27), 57 (30), 41 (26); HRMS: $m / z$ calcd for $\mathrm{C}_{31} \mathrm{H}_{36} \mathrm{O}_{4} \mathrm{Si}: 500.2383[M]^{+*}$; found: 500.2380; elemental analysis: calcd $(\%)$ for $\mathrm{C}_{31} \mathrm{H}_{36} \mathrm{O}_{4} \mathrm{Si}$ : C 74.36, $\mathrm{H}$ 7.25; found: C 74.56, H 7.53.

10a and 10b: Powdered $\mathrm{NaOH}$ (593 mg, $14.8 \mathrm{mmol}$ ), benzyltriethylammonium chloride (TEBAC; $11 \mathrm{mg}, 49 \mu \mathrm{mol})$, and $\mathrm{CHCl}_{3}(791 \mu \mathrm{L}$, $9.88 \mathrm{mmol})$ were added sequentially to a solution of the alkene $9(1.24 \mathrm{~g}$, $2.47 \mathrm{mmol})$ in $\mathrm{CH}_{2} \mathrm{Cl}_{2}(1.80 \mathrm{~mL})$ at $18^{\circ} \mathrm{C}$ under a nitrogen atmosphere. The resulting mixture was cooled in an ice bath (to control the ensuing exothermic reaction) and sonicated for $10 \mathrm{~min}$. The resulting mixture was filtered through a pad of celite, which was then washed with copious quantities of $\mathrm{CH}_{2} \mathrm{Cl}_{2}$. The combined filtrates were concentrated under reduced pressure to give a dark-brown oil, which was purified by flash chromatography (silica, ethyl acetate/hexane $=5: 95$ ) to give an approximately $2: 1$ mixture of $\mathbf{1 0} \mathbf{a}$ and $\mathbf{1 0 b}(1.31 \mathrm{~g}, 91 \%)$ as a viscous, amber-colored oil. $R_{\mathrm{f}}=0.1$ (ethyl acetate/hexane $=5: 95$ ); IR (film): $\tilde{v}=3071,2931$, 2892, 2858, 1503, 1486, 1428, 1382, 1239, 1110, 1043, 938, 824, 737, 703, $614,505 \mathrm{~cm}^{-1}$; ${ }^{1} \mathrm{H}$ NMR (300 MHz, $\mathrm{CDCl}_{3}$; major diastereoisomer): $\delta=$ 7.69-7.63 (complex m, 4H), 7.42-7.34 (complex m, 6H), 6.74 (s, 1 H), 6.62 $(\mathrm{s}, 1 \mathrm{H}), 5.90(\mathrm{~m}, 2 \mathrm{H}), 3.89(\mathrm{~m}, 2 \mathrm{H}), 3.80(\mathrm{~m}, 1 \mathrm{H}), 3.12(\mathrm{~s}, 3 \mathrm{H}), 2.90(\mathrm{~m}$, 2H), 2.46 (dd, $J=14.7,6.2 \mathrm{~Hz}, 1 \mathrm{H}), 2.35-1.95$ (complex m, 4H), $1.06 \mathrm{ppm}(\mathrm{s}, 9 \mathrm{H}) ;{ }^{13} \mathrm{C} \mathrm{NMR}\left(75 \mathrm{MHz}, \mathrm{CDCl}_{3}\right.$; major diastereoisomer): $\delta=146.9\left(\mathrm{C}_{\text {quat }}\right), 146.0\left(\mathrm{C}_{\text {quat }}\right), 135.7(\mathrm{CH}), 133.8\left(\mathrm{C}_{\text {quat }}\right), 132.7\left(\mathrm{C}_{\text {quat }}\right)$, $131.6\left(\mathrm{C}_{\text {quat }}\right), 129.7(\mathrm{CH}), 127.8(\mathrm{CH}), 109.8(\mathrm{CH}), 108.6(\mathrm{CH}), 101.1$ $\left(\mathrm{CH}_{2}\right), 85.2(\mathrm{CH}), 73.2\left(\mathrm{C}_{\text {quat }}\right), 64.8\left(\mathrm{CH}_{2}\right), 56.6\left(\mathrm{CH}_{3}\right), 47.8\left(\mathrm{C}_{\text {quat }}\right), 43.2$ $\left(\mathrm{CH}_{2}\right), 40.7(\mathrm{CH}), 35.8\left(\mathrm{CH}_{2}\right), 35.7\left(\mathrm{CH}_{2}\right), 27.0\left(\mathrm{CH}_{3}\right), 19.3 \mathrm{ppm}\left(\mathrm{C}_{\text {quat }}\right)$; MS (EI, $70 \mathrm{eV}): m / z(\%): 586,584$, and $582\left(3,12\right.$, and 16) $[M]^{+\cdot}, 460$ and 458 (23 and 60), 459 and 457 (69 and 100), 259 (13), 229 (23), 224 (28), 199 (18), 183 (11), 135 (13), 91 (10); HRMS: $\mathrm{m} / \mathrm{z}$ calcd for $\mathrm{C}_{32} \mathrm{H}_{36}{ }^{35} \mathrm{Cl}_{2} \mathrm{O}_{4} \mathrm{Si}: 582.1760[M]^{+\bullet}$; found: 582.1772 .

$11 \mathrm{a}$ and $11 \mathbf{b}$ : TBAF $(1.10 \mathrm{~mL}$ of a $1.0 \mathrm{~m}$ solution in THF, $1.10 \mathrm{mmol})$ was added dropwise to a magnetically stirred solution of a mixture of $\mathbf{1 0 a}$ and $10 \mathrm{~b}$ (ca. $2: 1 ; 496 \mathrm{mg}, 850 \mu \mathrm{mol})$ in THF $(10 \mathrm{~mL})$ at approximately $0^{\circ} \mathrm{C}$. The resulting mixture was warmed to $18^{\circ} \mathrm{C}$ and stirred at this temperature for $2.5 \mathrm{~h}$. $\mathrm{NH}_{4} \mathrm{Cl}(10 \mathrm{~mL}$ of a saturated aqueous solution) was then added, and the mixture was extracted with diethyl ether $(3 \times 30 \mathrm{~mL})$. The combined organic extracts were dried $\left(\mathrm{Na}_{2} \mathrm{SO}_{4}\right)$, filtered, and concentrated under reduced pressure to give an orange-brown oil, which was purified by flash chromatography (silica, ethyl acetate/hexane $=3: 7$ ) to give an approximately 2:1 mixture of $\mathbf{1 1}$ a and $\mathbf{1 1 b}(284 \mathrm{mg}, 97 \%)$ as a pale-amber oil. $R_{\mathrm{f}}=0.2$ (ethyl acetate/hexane $=3: 7$ ); IR (film): $\tilde{v}=3401$, 2931, 2894, 1503, 1486, 1383, 1238, 1097, 1041, 935, $842 \mathrm{~cm}^{-1} ;{ }^{1} \mathrm{H}$ NMR (300 MHz, $\mathrm{CDCl}_{3}$; major diastereoisomer): $\delta=6.84(\mathrm{~s}, 1 \mathrm{H}), 6.54(\mathrm{~s}, 1 \mathrm{H})$, $5.94(\mathrm{~m}, 2 \mathrm{H}), 3.95(\mathrm{~m}, 2 \mathrm{H}), 3.27(\mathrm{~s}, 3 \mathrm{H}), 2.88(\mathrm{~m}, 2 \mathrm{H}), 2.71-2.63$ (complex m, 1H), 2.48-2.24 (complex m, 3H), $2.17(\mathrm{~m}, 1 \mathrm{H}), 1.85 \mathrm{ppm}(\mathrm{m}$, $1 \mathrm{H})$; no signal was observed for the hydrogen atom of the hydroxy group; ${ }^{13} \mathrm{CNMR}$ (75 MHz, $\mathrm{CDCl}_{3}$; major diastereoisomer): $\delta=147.1$ $\left(\mathrm{C}_{\text {quat }}\right), 146.1\left(\mathrm{C}_{\text {quat }}\right), 132.1\left(\mathrm{C}_{\text {quat }}\right), 131.8\left(\mathrm{C}_{\text {quat }}\right), 109.0(\mathrm{CH}), 108.8(\mathrm{CH})$, 101.2 $\left(\mathrm{CH}_{2}\right), 85.2(\mathrm{CH}), 73.2\left(\mathrm{C}_{\text {quat }}\right), 63.1\left(\mathrm{CH}_{2}\right), 56.6\left(\mathrm{CH}_{3}\right), 47.8\left(\mathrm{C}_{\text {quat }}\right)$, $43.3\left(\mathrm{CH}_{2}\right), 40.7(\mathrm{CH}), 35.7\left(\mathrm{CH}_{2}\right), 35.5 \mathrm{ppm}\left(\mathrm{CH}_{2}\right)$; MS (EI, $\left.70 \mathrm{eV}\right): \mathrm{m} / z$ (\%): 348, 346, and $344\left(16,90\right.$, and 100) $[M]^{+}, 279$ and 277 (21 and 46), 265 and 263 (30 and 75), 243 and 241 (24 and 61), 233 (72), 226 and 224 (27 and 65), 213 and 211 (41 and 80), 212 and 210 (30 and 58), 190 (56), 177 (61), 115 (59); HRMS: $m / z$ calcd for $\mathrm{C}_{16} \mathrm{H}_{18}{ }^{35} \mathrm{Cl}_{2} \mathrm{O}_{4}: 344.0582[M]^{+\bullet}$; found: 344.0584 ; elemental analysis: calcd (\%) for $\mathrm{C}_{16} \mathrm{H}_{18} \mathrm{Cl}_{2} \mathrm{O}_{4}$ : C 55.67, $\mathrm{H}$ 5.26, $\mathrm{Cl} 20.54$; found: C 55.41, H 5.05, Cl 20.71.

12a and 12b: $\mathrm{Et}_{3} \mathrm{~N}(280 \mu \mathrm{L}, 2.00 \mathrm{mmol})$, DMAP $(10 \mathrm{mg}, 80 \mu \mathrm{mol})$, and $\mathrm{MsCl}(155 \mu \mathrm{L}, 2.00 \mathrm{mmol})$ were added sequentially to a magnetically stirred solution of the alcohols 11 a and $\mathbf{1 1 b}(\approx 2: 1 ; 276 \mathrm{mg}, 799 \mu \mathrm{mol})$ in
$\mathrm{CH}_{2} \mathrm{Cl}_{2}(8 \mathrm{~mL})$ at $0^{\circ} \mathrm{C}$ under a nitrogen atmosphere. The resulting mixture was warmed to $18^{\circ} \mathrm{C}$ and stirred at this temperature for $16 \mathrm{~h}$. $\mathrm{NaHCO}_{3}(8 \mathrm{~mL}$ of a saturated aqueous solution) was then added, and the mixture was extracted with $\mathrm{CH}_{2} \mathrm{Cl}_{2}(3 \times 20 \mathrm{~mL})$. The combined organic extracts were dried $\left(\mathrm{Na}_{2} \mathrm{SO}_{4}\right)$, filtered, and concentrated under reduced pressure to give an orange oil, which was purified by flash chromatography (silica, ethyl acetate/hexane $=1: 3 \rightarrow 1: 2$ ) to give an approximately $2: 1$ mixture of 12a and $\mathbf{1 2 b}(338 \mathrm{mg}, 100 \%)$ as a pale-yellow oil. $R_{\mathrm{f}}=0.1$ (ethyl acetate/hexane =1:3); IR (film): $\tilde{v}=2934,1504,1488,1355,1238$, 1174, 1105, 1041, $955 \mathrm{~cm}^{-1}$; ${ }^{1} \mathrm{H}$ NMR $\left(300 \mathrm{MHz}, \mathrm{CDCl}_{3}\right.$; major diastereoisomer): $\delta=6.76(\mathrm{~s}, 1 \mathrm{H}), 6.67(\mathrm{~s}, 1 \mathrm{H}), 5.94(\mathrm{~m}, 2 \mathrm{H}), 4.47(\mathrm{~m}, 2 \mathrm{H}), 3.88$ $(\mathrm{m}, 1 \mathrm{H}), 3.19(\mathrm{~s}, 3 \mathrm{H}), 3.06(\mathrm{~m}, 2 \mathrm{H}), 3.00(\mathrm{~s}, 3 \mathrm{H}), 2.63(\mathrm{dd}, J=14.7$, $6.2 \mathrm{~Hz}, 1 \mathrm{H}), 2.33 \mathrm{ppm}(\mathrm{m}, 2 \mathrm{H})$; the signals due to two hydrogen atoms were obscured; ${ }^{13} \mathrm{C} \mathrm{NMR}\left(75 \mathrm{MHz}, \mathrm{CDCl}_{3}\right.$; major diastereoisomer): $\delta=$ $147.3\left(\mathrm{C}_{\text {quat }}\right), 146.7\left(\mathrm{C}_{\text {quat }}\right), 132.0\left(\mathrm{C}_{\text {quat }}\right), 129.5\left(\mathrm{C}_{\text {quat }}\right), 109.0(\mathrm{CH}), 108.9$ $(\mathrm{CH}), 101.3\left(\mathrm{CH}_{2}\right), 85.3(\mathrm{CH}), 73.1\left(\mathrm{C}_{\text {quat }}\right), 69.8\left(\mathrm{CH}_{2}\right), 56.5\left(\mathrm{CH}_{3}\right), 47.6$ $\left(\mathrm{C}_{\text {quat }}\right), 43.1\left(\mathrm{CH}_{2}\right), 40.8(\mathrm{CH}), 37.5\left(\mathrm{CH}_{3}\right), 35.3\left(\mathrm{CH}_{2}\right), 32.2 \mathrm{ppm}\left(\mathrm{CH}_{2}\right)$; MS (EI, $70 \mathrm{eV}): m / z(\%): 426,424$, and $422(6,31$, and 44$)[M]^{+\cdot}, 389$ and 387 (7 and 17), 388 and 386 (13 and 11), 293 and 291 (26 and 70), 267, 265, and 263 (10,18, and 52), 261 and 259 (39 and 95), 255 (69), 235 and 233 (18 and 54), 225 (64), 224 (92), 223 (100), 211 (60), 210 (51), 152 (52), 115 (50), 57 (57), 43 (69); HRMS: $m / z$ calcd for $\mathrm{C}_{17} \mathrm{H}_{20}{ }^{35} \mathrm{Cl}_{2} \mathrm{O}_{6} \mathrm{~S}$ : $422.0358[M]^{+\bullet}$; found: 422.0360 .

13a and 13b: $\operatorname{LiN}_{3}(320 \mathrm{mg}, 6.53 \mathrm{mmol})$ was added to a magnetically stirred solution of the mesylates $12 \mathbf{a}$ and $\mathbf{1 2 b}(\approx 2: 1 ; 922 \mathrm{mg}, 2.18 \mathrm{mmol})$ in anhydrous DMF $(8 \mathrm{~mL})$, and the resulting mixture was stirred at $18^{\circ} \mathrm{C}$ under a nitrogen atmosphere for $48 \mathrm{~h}$. The reaction mixture was then diluted with diethyl ether $(30 \mathrm{~mL})$, and the organic phase was washed with water $(3 \times 10 \mathrm{~mL})$, then dried $\left(\mathrm{Na}_{2} \mathrm{SO}_{4}\right)$, filtered, and concentrated under reduced pressure to give a yellow oil. Purification by flash chromatography ( silica, ethyl acetate/hexane $=1: 9$ ) gave an approximately $2: 1$ mixture of $13 \mathbf{a}$ and $13 \mathbf{b}\left(757 \mathrm{mg}, 94 \%\right.$ ) as a pale-yellow oil. $R_{\mathrm{f}}=0.3$ (major diastereoisomer) and 0.2 (minor diastereoisomer; ethyl acetate/hexane $=$ 1:9); IR (film): $\tilde{v}=2932,2897,2099,1504,1487,1382,1240,1108,1042$, 935, $842 \mathrm{~cm}^{-1} ;{ }^{1} \mathrm{H}$ NMR (300 MHz, $\mathrm{CDCl}_{3}$; major diastereoisomer): $\delta=$ $6.74(\mathrm{~s}, 1 \mathrm{H}), 6.67(\mathrm{~s}, 1 \mathrm{H}), 5.94(\mathrm{~m}, 2 \mathrm{H}), 3.89(\mathrm{~m}, 1 \mathrm{H}), 3.56(\mathrm{~m}, 2 \mathrm{H}), 3.20$ $(\mathrm{s}, 3 \mathrm{H}), 2.91(\mathrm{t}, J=7.6 \mathrm{~Hz}, 2 \mathrm{H}), 2.64(\mathrm{dd}, J=14.7,6.2 \mathrm{~Hz}, 1 \mathrm{H}), 2.36-$ $2.10 \mathrm{ppm}$ (complex m, $4 \mathrm{H}) ;{ }^{13} \mathrm{C}$ NMR $\left(75 \mathrm{MHz}, \mathrm{CDCl}_{3}\right.$ ): major diastereoisomer: $\delta=147.3\left(\mathrm{C}_{\text {quat }}\right), 146.5\left(\mathrm{C}_{\text {quat }}\right), 131.7\left(\mathrm{C}_{\text {quat }}\right), 131.4\left(\mathrm{C}_{\text {quat }}\right), 109.0$ $(\mathrm{CH}), 108.8(\mathrm{CH}), 101.3\left(\mathrm{CH}_{2}\right), 85.3(\mathrm{CH}), 73.1\left(\mathrm{C}_{\text {quat }}\right), 56.6\left(\mathrm{CH}_{3}\right), 51.9$ $\left(\mathrm{CH}_{2}\right), 47.7\left(\mathrm{C}_{\text {quat }}\right), 43.1\left(\mathrm{CH}_{2}\right), 40.9(\mathrm{CH}), 35.5\left(\mathrm{CH}_{2}\right), 32.0 \mathrm{ppm}\left(\mathrm{CH}_{2}\right)$; minor diastereoisomer: $\delta=147.3\left(\mathrm{C}_{\text {quat }}\right), 146.3\left(\mathrm{C}_{\text {quat }}\right), 132.2\left(\mathrm{C}_{\text {quat }}\right), 131.6$ $\left(\mathrm{C}_{\text {quat }}\right), 109.2(\mathrm{CH}), 108.7(\mathrm{CH}), 101.4\left(\mathrm{CH}_{2}\right), 85.9(\mathrm{CH}), 74.5\left(\mathrm{C}_{\text {quat }}\right), 57.7$ $\left(\mathrm{CH}_{3}\right), 51.6\left(\mathrm{CH}_{2}\right), 45.8\left(\mathrm{C}_{\text {quat }}\right), 41.7\left(\mathrm{CH}_{2}\right), 38.1(\mathrm{CH}), 33.5\left(\mathrm{CH}_{2}\right)$, $32.0 \mathrm{ppm}\left(\mathrm{CH}_{2}\right)$; MS (EI, $\left.70 \mathrm{eV}\right): \mathrm{m} / z$ (\%): 373, 371, and $369(10,45$, and $62)[M]^{+\cdot}, 344,342$, and $340(2,10$, and 15$), 330,328$, and $326(5,24$, and 35), 314, 312, and 310 (8, 30, and 46), 212 and 210 (25 and 51), 189 (100); HRMS: $m / z$ calcd for $\mathrm{C}_{16} \mathrm{H}_{17}{ }^{35} \mathrm{Cl}_{2} \mathrm{~N}_{3} \mathrm{O}_{3}: 369.0647[M]^{+\cdot}$; found: 369.0629 ; elemental analysis: calcd (\%) for $\mathrm{C}_{16} \mathrm{H}_{17} \mathrm{Cl}_{2} \mathrm{~N}_{3} \mathrm{O}_{3}$ : C 51.91, $\mathrm{H} 4.63, \mathrm{Cl}$ 19.15, N 11.35; found: C 51.94, H 4.72, Cl 19.20, N 11.37.

15a and 15b: $\mathrm{PPh}_{3}(796 \mathrm{mg}, 3.03 \mathrm{mmol})$ was added to a magnetically stirred solution of the azides $13 \mathbf{a}$ and $\mathbf{1 3 b}(\approx 2: 1 ; 749 \mathrm{mg}, 2.02 \mathrm{mmol})$ in $\mathrm{THF} /$ water $(10: 3,13 \mathrm{~mL})$, and the resulting mixture was stirred at $18^{\circ} \mathrm{C}$ for $16 \mathrm{~h}$. The solvent was then removed under reduced pressure, and the residue, which contained a mixture of the amines $14 \mathbf{a}$ and $\mathbf{1 4} \mathbf{b}$, was taken up in ethyl acetate $(15 \mathrm{~mL})$, then dried $\left(\mathrm{Na}_{2} \mathrm{SO}_{4}\right)$, filtered, and concentrated under reduced pressure to give a pale-yellow, oily solid. A magnetically stirred solution of this material in THF $(20 \mathrm{~mL})$ under a nitrogen atmosphere was cooled to $0^{\circ} \mathrm{C}$ then treated with pyridine $(327 \mu \mathrm{L}$, $4.04 \mathrm{mmol})$ and allyl chloroformate $(429 \mu \mathrm{L}, 4.04 \mathrm{mmol})$. The reaction mixture was stirred at $0^{\circ} \mathrm{C}$ for approximately $10 \mathrm{~min}$, then warmed to $18^{\circ} \mathrm{C}$ and stirred at this temperature for $16 \mathrm{~h}$. The solvent was then removed under reduced pressure, and the residue taken up in diethyl ether $(80 \mathrm{~mL})$. The resulting solution was washed with water $(1 \times 20 \mathrm{~mL})$ and then brine $(1 \times 30 \mathrm{~mL})$, dried $\left(\mathrm{Na}_{2} \mathrm{SO}_{4}\right)$, filtered, and concentrated under reduced pressure to give a yellow oil. Purification by flash chromatography ( mixture of $\mathbf{1 5} \mathbf{a}$ and $\mathbf{1 5 b}(824 \mathrm{mg}, 95 \%$ from 13) as a pale-yellow, viscous oil. $R_{\mathrm{f}}=0.2$ (ethyl acetate/hexane $=1: 4$ ); IR (film): $\tilde{v}=3337,2933,1717$, 
1504, 1487, 1383, 1240, 1107, 1040, 992, 934, $841 \mathrm{~cm}^{-1} ;{ }^{1} \mathrm{H}$ NMR (300 MHz, $\mathrm{CDCl}_{3}$; major diastereoisomer): $\delta=6.66(\mathrm{~s}, 1 \mathrm{H}), 6.53(\mathrm{~s}, 1 \mathrm{H})$, $5.94(\mathrm{~m}, 2 \mathrm{H}), 5.89$ (partially obscured $\mathrm{m}, 1 \mathrm{H}), 5.25(\mathrm{~m}, 2 \mathrm{H}), 4.84$ (br s, $1 \mathrm{H}, \mathrm{NH}), 4.57(\mathrm{~d}, J=5.5 \mathrm{~Hz}, 2 \mathrm{H}), 3.88(\mathrm{~m}, 1 \mathrm{H}), 3.52-3.48$ (complex m, $2 \mathrm{H}$ ), 3.19 (s, 3H), 2.87-2.70 (complex m, 2H), 2.68-2.61 (complex m, $2 \mathrm{H}), 2.35-2.05 \mathrm{ppm}$ (complex m, 3H); ${ }^{13} \mathrm{C} \mathrm{NMR}\left(75 \mathrm{MHz}, \mathrm{CDCl}_{3}\right.$; major diastereoisomer): $\delta=156.2(\mathrm{CO}), 147.3\left(\mathrm{C}_{\text {quat }}\right), 146.2\left(\mathrm{C}_{\text {quat }}\right), 133.0(\mathrm{CH})$, $132.1\left(\mathrm{C}_{\text {quat }}\right), 131.7\left(\mathrm{C}_{\text {quat }}\right), 117.7\left(\mathrm{CH}_{2}\right), 108.7(\mathrm{CH}), 108.6(\mathrm{CH}), 101.2$ $\left(\mathrm{CH}_{2}\right), 85.3(\mathrm{CH}), 73.2\left(\mathrm{C}_{\text {quat }}\right), 65.5\left(\mathrm{CH}_{2}\right), 56.5\left(\mathrm{CH}_{3}\right), 47.8\left(\mathrm{C}_{\text {quat }}\right), 43.1$ $\left(\mathrm{CH}_{2}\right), 41.6\left(\mathrm{CH}_{2}\right), 40.7(\mathrm{CH}), 35.5\left(\mathrm{CH}_{2}\right), 32.8 \mathrm{ppm}\left(\mathrm{CH}_{2}\right)$; MS (EI, $70 \mathrm{eV}): m / z(\%): 431,429$, and $427\left(2,10\right.$, and 15) $[M]^{+\cdot}, 393$ and $391(4$ and 6), 344 and 342 (13 and 19), 328 and 326 (16 and 25), 320 and 318 (33 and 76), 41 (100); HRMS: $m / z$ calcd for $\mathrm{C}_{20} \mathrm{H}_{23}{ }^{35} \mathrm{Cl}_{2} \mathrm{NO}_{5}: 427.0953$ $[M]^{+*}$; found: 427.0953; elemental analysis: calcd (\%) for $\mathrm{C}_{20} \mathrm{H}_{23} \mathrm{Cl}_{2} \mathrm{NO}_{5}$ : C 56.08, H 5.41, Cl 16.55, N 3.27; found: C 56.33, H 5.58, Cl 16.19, N 3.23 .

16 and 17: LiHMDS ( $900 \mu \mathrm{L}$ of a $1.0 \mathrm{~m}$ solution in THF, $900 \mu \mathrm{mol})$ was added to a magnetically stirred solution of the gem-dichlorocyclopropanes 15a and 15b (ca. $2: 1 ; 350 \mathrm{mg}, 817 \mu \mathrm{mol}$ ) in anhydrous THF $(12 \mathrm{~mL})$ at $-20^{\circ} \mathrm{C}$ under an argon atmosphere. The reaction mixture was stirred at $-20^{\circ} \mathrm{C}$ for $10 \mathrm{~min}$, then warmed to $0^{\circ} \mathrm{C}$ and stirred at this temperature for a further $0.5 \mathrm{~h} . \mathrm{AgBF}_{4}(636 \mathrm{mg}, 3.27 \mu \mathrm{mol})$ was added in one portion, and the reaction mixture was stirred at $0^{\circ} \mathrm{C}$ for a further $10 \mathrm{~min}$, then warmed to $18^{\circ} \mathrm{C}$ and stirred at this temperature for $0.5 \mathrm{~h}$. The reaction mixture was then heated at $45^{\circ} \mathrm{C}$ for $4 \mathrm{~h}$, cooled, and filtered through a pad of celite. The filtrate was concentrated under reduced pressure to give a yellow solid. A magnetically stirred solution of this material in anhydrous THF $(10 \mathrm{~mL})$ was treated with dimedone $(573 \mathrm{mg}$, $4.09 \mathrm{mmol})$ and $\left[\mathrm{Pd}\left(\mathrm{PPh}_{3}\right)_{4}\right](172 \mathrm{mg}, 149 \mu \mathrm{mol})$, and the resulting mixture was stirred at $18^{\circ} \mathrm{C}$ under an argon atmosphere for $16 \mathrm{~h}$. The reaction mixture was then concentrated under reduced pressure to give a brown semisolid, which was subjected to flash chromatography (silica, $\mathrm{CHCl}_{3} / \mathrm{MeOH}=9: 1$ with $5 \% \quad v / v 880$ ammonia). The relevant fractions $\left(R_{\mathrm{f}}=0.7\left(\mathrm{CHCl}_{3} / \mathrm{MeOH}=9: 1\right.\right.$ with $5 \% \quad v / v 880$ ammonia $\left.)\right)$ were concentrated to give a mixture of the target amines and $\mathrm{Ph}_{3} \mathrm{P}(\mathrm{O})$. This mixture was purified further by flash chromatography (silica, methanol $/ \mathrm{CH}_{2} \mathrm{Cl}_{2}=$ $1: 99 \rightarrow 2.5: 97.5)$ to give $\mathbf{1 6}^{2}(65 \mathrm{mg}, 26 \%)$ as an orange oil and $\mathbf{1 7}(76 \mathrm{mg}$, $30 \%)$ as an orange foam. 16: $R_{\mathrm{f}}=0.1(1)$ (ethyl acetate/hexane $=2: 3$ ); IR (film): $\tilde{v}=2924,1502,1484,1384,1234,1089,1039,933 \mathrm{~cm}^{-1} ;{ }^{1} \mathrm{H}$ NMR $\left(300 \mathrm{MHz}, \mathrm{CDCl}_{3}\right): \delta=6.54(\mathrm{~s}, 1 \mathrm{H}), 6.53(\mathrm{~s}, 1 \mathrm{H}), 5.98(\mathrm{t}, J=4.1 \mathrm{~Hz}, 1 \mathrm{H})$, $5.90(\mathrm{~s}, 2 \mathrm{H}), 3.72(\mathrm{~m}, 1 \mathrm{H}), 3.32(\mathrm{~s}, 3 \mathrm{H}), 3.18(\mathrm{dd}, J=8.5,3.1 \mathrm{~Hz}, 2 \mathrm{H})$, $2.94(\mathrm{~m}, 2 \mathrm{H}), 2.62-2.54($ complex m, 3H) $, 2.46(\mathrm{~m}, 1 \mathrm{H}), 2.01(\mathrm{dd}, J=$ 14.2, $2.6 \mathrm{~Hz}, 1 \mathrm{H}) ;{ }^{13} \mathrm{C} \mathrm{NMR}\left(75 \mathrm{MHz}, \mathrm{CDCl}_{3}\right): \delta=146.4\left(\mathrm{C}_{\text {quat }}\right), 146.1$ $\left(\mathrm{C}_{\text {quat }}\right), 138.1\left(\mathrm{C}_{\text {quat }}\right), 132.8\left(\mathrm{C}_{\text {quat }}\right), 129.4\left(\mathrm{C}_{\text {quat }}\right), 124.5(\mathrm{CH}), 108.8(\mathrm{CH})$, $106.7(\mathrm{CH}), 100.9\left(\mathrm{CH}_{2}\right), 73.7(\mathrm{CH}), 60.5\left(\mathrm{C}_{\text {quat }}\right), 56.6\left(\mathrm{CH}_{3}\right), 40.9\left(\mathrm{CH}_{2}\right)$, $38.6\left(\mathrm{CH}_{2}\right), 30.9\left(\mathrm{CH}_{2}\right), 29.5 \mathrm{ppm}\left(\mathrm{CH}_{2}\right)$; MS (EI, $\left.70 \mathrm{eV}\right): \mathrm{m} / z(\%): 309$ and 307 (4 and 11) $[M]^{+*}, 294$ and 292 (2 and 5), 278 and 276 (13 and 30), 272 (6), 251 and 249 (31 and 75), 250 and 248 (55 and 100), 232 (58); HRMS: $m / z$ calcd for $\mathrm{C}_{16} \mathrm{H}_{18}{ }^{35} \mathrm{ClNO}_{3}: 307.0975$ [M] ${ }^{+\bullet}$; found: 307.0976 . 17: $R_{\mathrm{f}}=0.0(9)$ (ethyl acetate/hexane $=2: 3$ ); IR (film): $\tilde{v}=2930,1502$, 1483, 1382, 1233, 1095, 1038, $991 \mathrm{~cm}^{-1} ;{ }^{1} \mathrm{H}$ NMR (300 MHz, $\left.\mathrm{CDCl}_{3}\right): \delta=$ $6.55(\mathrm{~s}, 1 \mathrm{H}), 6.51(\mathrm{~s}, 1 \mathrm{H}), 5.97(\mathrm{dd}, J=6.3,2.2 \mathrm{~Hz}, 1 \mathrm{H}), 5.90(\mathrm{br} \mathrm{s}, 2 \mathrm{H})$, $3.72(\mathrm{~m}, 1 \mathrm{H}), 3.37(\mathrm{~s}, 3 \mathrm{H}), 3.13(\mathrm{~m}, 2 \mathrm{H}), 2.80-2.50$ (complex m, 4H), $2.16(\mathrm{~m}, 2 \mathrm{H}), 1.70 \mathrm{ppm}(\mathrm{m}, 1 \mathrm{H}) ;{ }^{13} \mathrm{C} \mathrm{NMR}\left(75 \mathrm{MHz}, \mathrm{CDCl}_{3}\right): \delta=146.3$ $\left(\mathrm{C}_{\text {quat }}\right), 137.7\left(\mathrm{C}_{\text {quat }}\right), 132.7\left(\mathrm{C}_{\text {quat }}\right), 129.2\left(\mathrm{C}_{\text {quat }}\right), 126.1(\mathrm{CH}), 108.4(\mathrm{CH})$, $106.8(\mathrm{CH}), 100.8\left(\mathrm{CH}_{2}\right), 72.6(\mathrm{CH}), 62.6\left(\mathrm{C}_{\text {quat }}\right), 56.0\left(\mathrm{CH}_{3}\right), 43.4\left(\mathrm{CH}_{2}\right)$, $39.2\left(\mathrm{CH}_{2}\right), 32.8\left(\mathrm{CH}_{2}\right), 30.0 \mathrm{ppm}\left(\mathrm{CH}_{2}\right)$; one signal due to a quaternary carbon atom was obscured and may overlap with another signal; MS (EI, $70 \mathrm{eV}): m / z$ (\%): 309 and 307 (16 and 39) $[\mathrm{M}]^{+}, 294$ and 292 (4 and 10), 278 and 276 (24 and 50), 272 (11), 251 and 249 (57 and 85), 250 and 248 (77 and 100), 232 (54); HRMS: $m / z$ calcd for $\mathrm{C}_{16} \mathrm{H}_{18}{ }^{35} \mathrm{ClNO}_{3}: 307.0975$ $[M]^{+\bullet}$; found: 307.0973 .

18: Ethylene oxide $(\approx 2 \mathrm{~mL}, \approx 40 \mathrm{mmol}$ ) was added to a solution of the amine $16(63 \mathrm{mg}, 205 \mu \mathrm{mol})$ in anhydrous $\mathrm{MeOH}(1.0 \mathrm{~mL})$ at $0^{\circ} \mathrm{C}$ under a nitrogen atmosphere. The reaction vessel was sealed, and the mixture was stirred magnetically at $45^{\circ} \mathrm{C}$ for $24 \mathrm{~h}$. The reaction mixture was then

2 This material was contaminated with a small amount $(\leq 5 \%)$ of triphenylphosphine oxide. cooled to $18^{\circ} \mathrm{C}$, the vessel was opened, and the solvent was removed under reduced pressure to give a yellow oil. Purification by flash chromatography (silica, ethyl acetate/hexane =2:3) afforded $\mathbf{1 8}(41 \mathrm{mg}, 58 \%)$, which crystallized as pale-pink crystals from $\mathrm{CH}_{2} \mathrm{Cl}_{2} /$ hexane. $R_{\mathrm{f}}=0.2$ (ethyl acetate/hexane $=2: 3)$; decomp. from $109^{\circ} \mathrm{C} ;[\alpha]_{\mathrm{D}}^{20}=-91.3(c=1.0 \mathrm{~g}$ $\left.(100 \mathrm{~mL})^{-1}, \mathrm{CHCl}_{3}\right)$; IR (disk): $\tilde{v}=3450$ (br), 2924, 1503, 1486, 1384, 1234, 1102, 1037, $932 \mathrm{~cm}^{-1} ;{ }^{1} \mathrm{H}$ NMR $\left(300 \mathrm{MHz}, \mathrm{CDCl}_{3}\right): \delta=6.66(\mathrm{~s}, 1 \mathrm{H})$, $6.59(\mathrm{~s}, 1 \mathrm{H}), 6.22(\mathrm{dd}, J=7.1,2.2,1 \mathrm{H}), 5.93(\mathrm{~d}, J=1.5 \mathrm{~Hz}, 1 \mathrm{H}), 5.92(\mathrm{~d}$, $J=1.5 \mathrm{~Hz}, 1 \mathrm{H}), 3.81-3.65$ (complex m, 2H), $3.58(\mathrm{~m}, 1 \mathrm{H}), 3.29(\mathrm{~s}, 3 \mathrm{H})$, 3.08-2.80 (complex m, 5 H), 2.79-2.64 (complex m, 2H), 2.44 (dt, $J=12.7$, $2.7 \mathrm{~Hz}, 1 \mathrm{H}), 2.33(\mathrm{~m}, 1 \mathrm{H}), 2.09(\mathrm{~m}, 1 \mathrm{H}), 1.75 \mathrm{ppm}(\mathrm{m}, 1 \mathrm{H}) ;{ }^{13} \mathrm{C}$ NMR $\left(75 \mathrm{MHz}, \mathrm{CDCl}_{3}\right): \delta=146.6\left(\mathrm{C}_{\text {quat }}\right), 145.8\left(\mathrm{C}_{\text {quat }}\right), 136.2\left(\mathrm{C}_{\text {quat }}\right), 132.9$ $\left(\mathrm{C}_{\text {quat }}\right), 128.9(\mathrm{CH}), 127.6\left(\mathrm{C}_{\text {quat }}\right), 109.0(\mathrm{CH}), 106.8(\mathrm{CH}), 101.1\left(\mathrm{CH}_{2}\right)$, $72.9(\mathrm{CH}), 67.2\left(\mathrm{C}_{\text {quat }}\right), 58.4\left(\mathrm{CH}_{2}\right), 56.3\left(\mathrm{CH}_{3}\right), 51.6\left(\mathrm{CH}_{2}\right), 41.8\left(\mathrm{CH}_{2}\right)$, $37.1\left(\mathrm{CH}_{2}\right), 32.1\left(\mathrm{CH}_{2}\right), 29.6 \mathrm{ppm}\left(\mathrm{CH}_{2}\right)$; MS (EI, $\left.70 \mathrm{eV}\right): m / z(\%): 353$ and 351 ( 1 and 4$)[M]^{+*}, 338$ and $336(<1$ and 3$), 323$ and 321 (10 and 30), 322 and 320 (47 and 100), 316 (5), 295 and 293 (10 and 30); HRMS: $m / z$ calcd for $\mathrm{C}_{18} \mathrm{H}_{22}{ }^{35} \mathrm{ClNO}_{4}: 351.1237[M]^{+\cdot}$; found: 351.1240 .

19: $\mathrm{PPh}_{3}(155 \mathrm{mg}, 591 \mu \mathrm{mol})$, imidazole $(40 \mathrm{mg}, 591 \mu \mathrm{mol})$, and iodine $(113 \mathrm{mg}, 443 \mu \mathrm{mol})$ were added to a magnetically stirred solution of the alcohol $18(52 \mathrm{mg}, 148 \mu \mathrm{mol})$ in anhydrous toluene $(3 \mathrm{~mL})$ under a nitrogen atmosphere. The resulting mixture was stirred at $18^{\circ} \mathrm{C}$ for $16 \mathrm{~h}$, then $\mathrm{Na}_{2} \mathrm{~S}_{2} \mathrm{O}_{3}$ (2 mL of a saturated aqueous solution) was added, and stirring was continued for $5 \mathrm{~min}$. The mixture was extracted with ethyl acetate $(3 \times 20 \mathrm{~mL})$, and the combined organic extracts were dried $\left(\mathrm{Na}_{2} \mathrm{SO}_{4}\right)$, filtered, and concentrated under reduced pressure to give a brown oil. $\mathrm{Pu}$ rification by flash chromatography (silica, ethyl acetate/hexane $=4: 96$ ) gave $19(51 \mathrm{mg}, 75 \%)$ as a white powder. $R_{\mathrm{f}}=0.1$ (ethyl acetate/hexane $=$ $4: 96)$; decomp. from $121^{\circ} \mathrm{C} ;[\alpha]_{\mathrm{D}}^{20}=-60.1\left(c=1.0 \mathrm{~g}(100 \mathrm{~mL})^{-1}, \mathrm{CHCl}_{3}\right)$; IR (film): $\tilde{v}=2922,2837,1502,1484,1384,1234,1130,1095,1039,931$, $731 \mathrm{~cm}^{-1} ;{ }^{1} \mathrm{H}$ NMR $\left(300 \mathrm{MHz}, \mathrm{CDCl}_{3}\right): \delta=6.62(\mathrm{~s}, 1 \mathrm{H}), 6.58(\mathrm{~s}, 1 \mathrm{H}), 6.19$ $(\mathrm{dd}, J=6.6,2.2 \mathrm{~Hz}, 1 \mathrm{H}), 5.92(\mathrm{~d}, J=1.4 \mathrm{~Hz}, 1 \mathrm{H}), 5.91(\mathrm{~d}, J=1.4 \mathrm{~Hz}, 1 \mathrm{H})$, $3.65(\mathrm{~m}, 1 \mathrm{H}), 3.27(\mathrm{~s}, 3 \mathrm{H}), 3.34-3.16$ (partially obscured $\mathrm{m}, 2 \mathrm{H}), 3.08-$ 2.97 (complex m, 4H), 2.76-2.61 (complex m, 3H), 2.32-2.26 (complex m, $1 \mathrm{H}), 2.12(\mathrm{dd}, J=9.8,2.2 \mathrm{~Hz}, 1 \mathrm{H}), 1.75 \mathrm{ppm}(\mathrm{m}, 1 \mathrm{H}) ;{ }^{13} \mathrm{C} \mathrm{NMR}$ $\left(75 \mathrm{MHz}, \mathrm{CDCl}_{3}\right): \delta=146.6\left(\mathrm{C}_{\text {quat }}\right), 145.7\left(\mathrm{C}_{\text {quat }}\right), 136.4\left(\mathrm{C}_{\text {quat }}\right), 132.5$ $\left(\mathrm{C}_{\text {quat }}\right), 128.4(\mathrm{CH}), 127.7\left(\mathrm{C}_{\text {quat }}\right), 109.1(\mathrm{CH}), 106.7(\mathrm{CH}), 101.0\left(\mathrm{CH}_{2}\right)$, $72.7(\mathrm{CH}), 67.2\left(\mathrm{C}_{\text {quat }}\right), 56.2\left(\mathrm{CH}_{3}\right), 54.6\left(\mathrm{CH}_{2}\right), 43.7\left(\mathrm{CH}_{2}\right), 38.2\left(\mathrm{CH}_{2}\right)$, $32.2\left(\mathrm{CH}_{2}\right), 29.7\left(\mathrm{CH}_{2}\right), 4.4 \mathrm{ppm}\left(\mathrm{CH}_{2}\right)$; MS (EI, $\left.70 \mathrm{eV}\right): \mathrm{m} / z(\%): 463$ and 461 (15 and 37) $[M]^{+*}, 433$ and 431 (8 and 22), 432 and 430 (35 and 72), 426 (66), 405 and 403 (45 and 84), 336 and 334 (46 and 84), 278 and 276 (75 and 100), 240 (90); HRMS: $m / z$ calcd for $\mathrm{C}_{18} \mathrm{H}_{21}{ }^{35} \mathrm{ClINO}_{3}$ : $461.0255[M]^{+\cdot}$; found: 461.0254.

(-)-1: $\mathrm{Bu}_{3} \mathrm{SnH}(56 \mu \mathrm{L}, 210 \mu \mathrm{mol})$ was added over a period of $3.5 \mathrm{~h}$ to a magnetically stirred solution of AIBN $(5 \mathrm{mg}, 33 \mu \mathrm{mol}$; added in three equal aliquots over $2 \mathrm{~h}$ ) and the iodide $19(44 \mathrm{mg}, 95 \mu \mathrm{mol})$ in anhydrous toluene $(10 \mathrm{~mL})$ at $80^{\circ} \mathrm{C}$ under an argon atmosphere. Once the addition of $\mathrm{Bu}_{3} \mathrm{SnH}$ was complete, the reaction mixture was cooled to $18^{\circ} \mathrm{C}$ and concentrated under reduced pressure. The residue was purified by flash chromatography (silica, hexane $\rightarrow \mathrm{CH}_{2} \mathrm{Cl}_{2} /$ hexane $=1: 4 \rightarrow \mathrm{CH}_{2} \mathrm{Cl}_{2} /$ hexane $=$ $1: 1 \rightarrow \mathrm{CH}_{2} \mathrm{Cl}_{2} \rightarrow$ methanol $/ \mathrm{CH}_{2} \mathrm{Cl}_{2}=2.5: 97.5 \rightarrow$ methanol $/ \mathrm{CH}_{2} \mathrm{Cl}_{2}=5: 95 \rightarrow$ methanol $/ \mathrm{CH}_{2} \mathrm{Cl}_{2}=1: 9 \rightarrow$ methanol $\left./ \mathrm{CH}_{2} \mathrm{Cl}_{2}=1: 4\right)$ to give $(-)-\mathbf{1}(25 \mathrm{mg}$, $89 \%)$ as a colorless oil. $R_{\mathrm{f}}=0.4\left(\right.$ methanol/ $\left.\mathrm{CH}_{2} \mathrm{Cl}_{2}=1: 9\right) ;[\alpha]_{\mathrm{D}}^{20}=-187$ $\left(c=1.2 \mathrm{~g}(100 \mathrm{~mL})^{-1}\right.$, ethanol or $\left.\mathrm{CHCl}_{3}\right)$; IR (film): $\tilde{v}=2924,1502,1481$, 1371, 1231, 1101, 1080, 1036, 934, $875 \mathrm{~cm}^{-1} ;{ }^{1} \mathrm{H}$ NMR (300 MHz, $\mathrm{CDCl}_{3}$ ): $\delta=6.58(\mathrm{~s}, 1 \mathrm{H}), 6.57(\mathrm{~s}, 1 \mathrm{H}), 5.90(\mathrm{~d}, J=1.6 \mathrm{~Hz}, 1 \mathrm{H}), 5.89(\mathrm{~d}, J=1.6 \mathrm{~Hz}$, $1 \mathrm{H}), 5.61(\mathrm{~m}, 1 \mathrm{H}), 3.73(\mathrm{~m}, 1 \mathrm{H}), 3.52(\mathrm{~m}, 1 \mathrm{H}), 3.28(\mathrm{~s}, 3 \mathrm{H}), 3.13(\mathrm{ddd}$, $J=14.4,8.0,2.4 \mathrm{~Hz}, 1 \mathrm{H}), 3.00(\mathrm{~m}, 1 \mathrm{H}), 2.97(\mathrm{~m}, 1 \mathrm{H}), 2.73-2.67$ (complex m, $2 \mathrm{H}), 2.60(\mathrm{dd}, J=16.8,5.6 \mathrm{~Hz}, 1 \mathrm{H}), 2.43(\mathrm{~m}, 1 \mathrm{H}), 2.29(\mathrm{dd}, J=$ 11.2, $4.0 \mathrm{~Hz}, 1 \mathrm{H}), 2.21(\mathrm{~m}, 1 \mathrm{H}), 2.10(\mathrm{~m}, 1 \mathrm{H}), 1.63 \mathrm{ppm}(\mathrm{br} \mathrm{t}, J=11.2 \mathrm{~Hz}$, $1 \mathrm{H}) ;{ }^{13} \mathrm{C}$ NMR $\left(75 \mathrm{MHz}, \mathrm{CDCl}_{3}\right): \delta=146.5\left(\mathrm{C}_{\text {quat }}\right), 145.4\left(\mathrm{C}_{\text {quat }}\right), 140.6$ $\left(\mathrm{C}_{\text {quat }}\right), 130.9\left(\mathrm{C}_{\text {quat }}\right), 126.6\left(\mathrm{C}_{\text {quat }}\right), 118.5(\mathrm{CH}), 109.2(\mathrm{CH}), 107.9(\mathrm{CH})$, $100.8\left(\mathrm{CH}_{2}\right), 73.7(\mathrm{CH}), 64.7\left(\mathrm{C}_{\text {quat }}\right), 56.2\left(\mathrm{CH}_{3}\right), 47.1\left(\mathrm{CH}_{2}\right), 41.7\left(\mathrm{CH}_{2}\right)$, $40.8\left(\mathrm{CH}_{2}\right), 31.9\left(\mathrm{CH}_{2}\right), 27.1\left(\mathrm{CH}_{2}\right), 22.5 \mathrm{ppm}\left(\mathrm{CH}_{2}\right) ; \mathrm{MS}(\mathrm{EI}, 70 \mathrm{eV}): \mathrm{m} / z$ (\%): 299 (35) $\left[\mathrm{M}^{+} \cdot\right.$, 298 (10), 284 (6), 269 (20), 268 (54), 242 (80), 241 (95), 240 (100); HRMS: $m / z$ calcd for $\mathrm{C}_{18} \mathrm{H}_{21} \mathrm{NO}_{3}: 299.1521[M]^{+\cdot}$; found: 299.1527. 
20: Ethylene oxide $(\approx 2 \mathrm{~mL}, \approx 40 \mathrm{mmol})$ was added to a solution of the amine $17(114 \mathrm{mg}, 370 \mu \mathrm{mol})$ in anhydrous $\mathrm{MeOH}(1.0 \mathrm{~mL})$ at $0^{\circ} \mathrm{C}$ under a nitrogen atmosphere. The reaction vessel was sealed, and the mixture was stirred magnetically at $45^{\circ} \mathrm{C}$ for $48 \mathrm{~h}$. The reaction mixture was then cooled to $18^{\circ} \mathrm{C}$, the vessel was opened, and the solvent was removed under reduced pressure to give an orange oil. Purification by flash chromatography (silica, ethyl acetate/hexane =2:3) afforded 20 (103 mg, $79 \%$ ) as a white foam. $R_{\mathrm{f}}=0.1$ (ethyl acetate/hexane $=2: 3$ ); $[\alpha]_{\mathrm{D}}^{20}=$ $+124.9\left(c=2.0 \mathrm{~g}(100 \mathrm{~mL})^{-1}, \mathrm{CHCl}_{3}\right)$; IR (film): $\tilde{v}=3466$ (br), 2928, 2824, $1503,1485,1388,1233,1130,1100,1039,985,932,847,730 \mathrm{~cm}^{-1}$; ${ }^{1} \mathrm{H}$ NMR $\left(300 \mathrm{MHz}, \mathrm{CDCl}_{3}\right): \delta=6.59(\mathrm{~s}, 1 \mathrm{H}), 6.49(\mathrm{~s}, 1 \mathrm{H}), 6.20(\mathrm{dd}, J=$ 6.5, $2.5 \mathrm{~Hz}, 1 \mathrm{H}$ ), 5.90 (br s, 2H), 3.76-3.67 (complex m, 2H), 3.56 (m, $1 \mathrm{H}), 3.33(\mathrm{~s}, 3 \mathrm{H}), 3.11-2.67$ (complex m, 7H), 2.59-2.47 (complex m, $2 \mathrm{H}), 2.19(\mathrm{~m}, 1 \mathrm{H}), 1.78 \mathrm{ppm}(\mathrm{dd}, J=14.5,1.8 \mathrm{~Hz}, 1 \mathrm{H}) ;{ }^{13} \mathrm{C} \mathrm{NMR}$ $\left(75 \mathrm{MHz}, \mathrm{CDCl}_{3}\right): \delta=146.5\left(\mathrm{C}_{\text {quat }}\right), 146.4\left(\mathrm{C}_{\text {quat }}\right), 137.1\left(\mathrm{C}_{\text {quat }}\right), 133.8$ $\left(\mathrm{C}_{\text {quat }}\right), 128.6\left(\mathrm{C}_{\text {quat }}\right), 128.2(\mathrm{CH}), 107.7(\mathrm{CH}), 106.9(\mathrm{CH}), 101.0\left(\mathrm{CH}_{2}\right)$, $73.0(\mathrm{CH}), 67.3\left(\mathrm{C}_{\text {quat }}\right), 58.0\left(\mathrm{CH}_{2}\right), 56.2\left(\mathrm{CH}_{3}\right), 52.2\left(\mathrm{CH}_{2}\right), 40.6\left(\mathrm{CH}_{2}\right)$, $39.5\left(\mathrm{CH}_{2}\right), 32.2\left(\mathrm{CH}_{2}\right), 29.9 \mathrm{ppm}\left(\mathrm{CH}_{2}\right) ; \mathrm{MS}(\mathrm{EI}, 70 \mathrm{eV}): \mathrm{m} / z(\%): 353$ and 351 (20 and 45) $[M]^{+\cdot}, 338$ and 336 (5 and 25), 323 and 321 (28 and 62), 322 and 320 (80 and 100), 295 and 293 (50 and 84), 258 (86), 224 (70), 214 (86); HRMS: $m / z$ calcd for $\mathrm{C}_{18} \mathrm{H}_{22}{ }^{35} \mathrm{ClNO}_{4}$ : $351.1237[M]^{+\bullet}$; found: 351.1228

21: $\mathrm{PPh}_{3}(149 \mathrm{mg}, 568 \mu \mathrm{mol})$, imidazole $(39 \mathrm{mg}, 568 \mu \mathrm{mol})$, and iodine $(108 \mathrm{mg}, 426 \mu \mathrm{mol})$ were added to a magnetically stirred solution of the alcohol $20(50 \mathrm{mg}, 142 \mu \mathrm{mol})$ in anhydrous toluene $(3 \mathrm{~mL})$. The resulting mixture was stirred at $18^{\circ} \mathrm{C}$ under a nitrogen atmosphere for $16 \mathrm{~h}$, then $\mathrm{Na}_{2} \mathrm{~S}_{2} \mathrm{O}_{3}$ (2 $\mathrm{mL}$ of a saturated aqueous solution) was added, and stirring was continued for $5 \mathrm{~min}$. The mixture was extracted with ethyl acetate $(3 \times 20 \mathrm{~mL})$, and the combined organic extracts were dried $\left(\mathrm{Na}_{2} \mathrm{SO}_{4}\right)$, filtered, and concentrated under reduced pressure to give a brown oil. $\mathrm{Pu}-$ rification by flash chromatography (silica, ethyl acetate/hexane $=5: 95$ ) gave $21(52 \mathrm{mg}, 79 \%)$ as an off-white foam. $R_{\mathrm{f}}=0.1$ (ethyl acetate/ hexane $=5: 95) ;[\alpha]_{\mathrm{D}}^{20}=+63.2\left(c=1.0 \mathrm{~g}(100 \mathrm{~mL})^{-1}, \mathrm{CHCl}_{3}\right) ; \mathrm{IR}($ film $): \tilde{v}=$ 2927, 2823, 1502, 1484, 1387, 1234, 1134, 1107, 1092, 1039, 986, 931, $852 \mathrm{~cm}^{-1} ;{ }^{1} \mathrm{H}$ NMR $\left(300 \mathrm{MHz}, \mathrm{CDCl}_{3}\right): \delta=6.58(\mathrm{~s}, 1 \mathrm{H}), 6.48(\mathrm{~s}, 1 \mathrm{H}), 6.19$ $(\mathrm{dd}, J=6.3,2.6 \mathrm{~Hz}, 1 \mathrm{H}), 5.89(\mathrm{~s}, 2 \mathrm{H}), 3.68(\mathrm{~m}, 1 \mathrm{H}), 3.34(\mathrm{~s}, 3 \mathrm{H}), 3.23$ 3.15 (complex m, 3H), 2.99-2.55 (complex m, 7H), $2.18(\mathrm{~m}, 1 \mathrm{H})$, $1.77 \mathrm{ppm}(\mathrm{dd}, J=14.3,11.4 \mathrm{~Hz}, 1 \mathrm{H}) ;{ }^{13} \mathrm{C} \mathrm{NMR}\left(75 \mathrm{MHz}, \mathrm{CDCl}_{3}\right): \delta=$ $146.4\left(\mathrm{C}_{\text {quat }}\right), 146.3\left(\mathrm{C}_{\text {quat }}\right), 137.2\left(\mathrm{C}_{\text {quat }}\right), 133.8\left(\mathrm{C}_{\text {quat }}\right), 128.5\left(\mathrm{C}_{\text {quat }}\right), 128.0$ $(\mathrm{CH}), 107.8(\mathrm{CH}), 106.9(\mathrm{CH}), 100.9\left(\mathrm{CH}_{2}\right), 73.0(\mathrm{CH}), 67.1\left(\mathrm{C}_{\text {quat }}\right), 56.2$ $\left(\mathrm{CH}_{3}\right), 54.3\left(\mathrm{CH}_{2}\right), 42.6\left(\mathrm{CH}_{2}\right), 40.9\left(\mathrm{CH}_{2}\right), 32.4\left(\mathrm{CH}_{2}\right), 29.8\left(\mathrm{CH}_{2}\right)$, $4.9 \mathrm{ppm}\left(\mathrm{CH}_{2}\right)$; MS (EI, $\left.70 \mathrm{eV}\right): \mathrm{m} / z(\%): 463$ and $461(6$ and 16$)[\mathrm{M}]^{+*}$, 432 and 430 (14 and 37), 426 (29), 405 and 403 (18 and 50), 336 and 334 (20 and 53), 278 and 276 (41 and 100), 240 (56), 73 (52), 69 (70), 43 (75); HRMS: $m / z$ calcd for $\mathrm{C}_{18} \mathrm{H}_{21}{ }^{35} \mathrm{ClINO}_{3}: 461.0255[M]^{+\cdot}$; found: 461.0253 . 3-epi-(+)-1: $\mathrm{Bu}_{3} \mathrm{SnH}(67 \mu \mathrm{L}, 248 \mu \mathrm{mol})$ was added over a period of $3.5 \mathrm{~h}$ to a magnetically stirred solution of AIBN $(5 \mathrm{mg}, 33 \mu \mathrm{mol}$; added in three equal aliquots over $2 \mathrm{~h}$ ) and the iodide $21(52 \mathrm{mg}, 113 \mu \mathrm{mol})$ in anhydrous toluene $(11 \mathrm{~mL})$ at $80^{\circ} \mathrm{C}$ under an argon atmosphere. Once the addition of $\mathrm{Bu}_{3} \mathrm{SnH}$ was complete, the reaction mixture was cooled to $18^{\circ} \mathrm{C}$ and concentrated under reduced pressure. The residue was purified by flash chromatography (silica, hexane $\rightarrow \mathrm{CH}_{2} \mathrm{Cl}_{2} /$ hexane $=1: 4 \rightarrow \mathrm{CH}_{2} \mathrm{Cl}_{2} /$ hexane $=1: 1 \rightarrow \mathrm{CH}_{2} \mathrm{Cl}_{2} \rightarrow$ methanol $/ \mathrm{CH}_{2} \mathrm{Cl}_{2}=2.5: 97.5 \rightarrow$ methanol $/ \mathrm{CH}_{2} \mathrm{Cl}_{2}=$ $5: 95 \rightarrow$ methanol/ $\mathrm{CH}_{2} \mathrm{Cl}_{2}=1: 9 \rightarrow$ methanol/ $\left.\mathrm{CH}_{2} \mathrm{Cl}_{2}=1: 4\right)$ to give 3-epi-(+)$1(34 \mathrm{mg}, 100 \%)$ as a pale-yellow semisolid. $R_{\mathrm{f}}=0.4\left(\right.$ methanol $/ \mathrm{CH}_{2} \mathrm{Cl}_{2}=$ $1: 9) ;[\alpha]_{\mathrm{D}}^{20}=+204\left(c=1.0 \mathrm{~g}(100 \mathrm{~mL})^{-1}, \mathrm{CHCl}_{3}\right)$; IR (film): $\tilde{v}=2927,1502$, 1481, 1376, 1230, 1099, 1039, 933, $864 \mathrm{~cm}^{-1} ;{ }^{1} \mathrm{H} \mathrm{NMR}\left(800 \mathrm{MHz}, \mathrm{CDCl}_{3}\right)$ : $\delta=6.66(\mathrm{~s}, 1 \mathrm{H}), 6.48(\mathrm{~s}, 1 \mathrm{H}), 5.86(\mathrm{~d}, J=1.6 \mathrm{~Hz}, 1 \mathrm{H}), 5.86(\mathrm{~d}, J=1.6 \mathrm{~Hz}$, $1 \mathrm{H}), 5.69(\mathrm{~m}, 1 \mathrm{H}), 3.57(\mathrm{~m}, 1 \mathrm{H}), 3.50(\mathrm{~m}, 1 \mathrm{H}), 3.20(\mathrm{~s}, 3 \mathrm{H}), 3.14(\mathrm{~m}$, $1 \mathrm{H}), 3.10(\mathrm{~m}, 1 \mathrm{H}), 3.03-2.98$ (complex m, 1 H), $2.79(\mathrm{~m}, 1 \mathrm{H}), 2.55-2.52$ (complex m, 2H), $2.41(\mathrm{~m}, 1 \mathrm{H}), 2.34(\mathrm{~m} \mathrm{1H}), 2.22-2.18$ (complex m, $2 \mathrm{H}), 1.86 \mathrm{ppm}(\mathrm{dd}, J=14.4,5.6 \mathrm{~Hz}, 1 \mathrm{H}) ;{ }^{13} \mathrm{C} \mathrm{NMR}\left(75 \mathrm{MHz}, \mathrm{CDCl}_{3}\right)$ : $\delta=146.5\left(\mathrm{C}_{\text {quat }}\right), 146.3\left(\mathrm{C}_{\text {quat }}\right), 141.4\left(\mathrm{C}_{\text {quat }}\right), 132.3\left(\mathrm{C}_{\text {quat }}\right), 125.7\left(\mathrm{C}_{\text {quat }}\right)$, $119.0(\mathrm{CH}), 108.1(\mathrm{CH}), 107.3(\mathrm{CH}), 100.8\left(\mathrm{CH}_{2}\right), 74.8(\mathrm{CH}), 64.7(\mathrm{C})$, $55.9\left(\mathrm{CH}_{3}\right), 46.4\left(\mathrm{CH}_{2}\right), 40.9\left(\mathrm{CH}_{2}\right), 40.0\left(\mathrm{CH}_{2}\right), 30.7\left(\mathrm{CH}_{2}\right), 27.5\left(\mathrm{CH}_{2}\right)$, $21.7 \mathrm{ppm}\left(\mathrm{CH}_{2}\right)$; MS (EI, $\left.70 \mathrm{eV}\right): \mathrm{m} / z(\%): 299(26)[M]^{+*}, 298$ (6), 284 (5), 269 (18), 268 (44), 242 (54), 241 (83), 240 (100); HRMS: $m / z$ calcd for $\mathrm{C}_{18} \mathrm{H}_{21} \mathrm{NO}_{3}: 299.1521[M]^{+*}$; found: 299.1519 .

\section{Crystallography}

Images were measured on a Nonius Kappa $\mathrm{CCD}$ diffractometer $\left(\mathrm{Mo}_{\mathrm{K} \alpha}\right.$, graphite monochromator, $\lambda=0.71073 \AA$ ), and data were extracted by using the DENZO package. ${ }^{[15]}$ The structure was solved by direct methods (SIR92). ${ }^{[16]}$ The structure of compound $\mathbf{1 8}$ was refined by using the CRYSTALS program package. ${ }^{[17]}$ CCDC-640224 contains the supplementary crystallographic data for this paper. These data can be obtained free-of-charge from The Cambridge Crystallographic Data Centre at http://www.ccdc.cam.ac.uk/data_request/cif.

Crystal data for 18: $\mathrm{C}_{18} \mathrm{H}_{22} \mathrm{ClNO}_{4}, M_{\mathrm{r}}=351.82, T=200(1) \mathrm{K}$, monoclinic, space group $P 2_{1}, Z=2, a=8.9545(2), b=7.4385(2), c=12.6365(3) \AA, \beta=$ 93.1564(13) $)^{\circ}, \quad V=840.42(4) \AA^{3}, \quad D_{x}=1.390 \mathrm{~g} \mathrm{~cm}^{-3}, \quad 3809$ unique data $\left(2 \theta_{\max }=55^{\circ}\right), 3556$ with $I>3.0 \sigma(I), R=0.0269, R w=0.0316, S=1.0524$, Flack parameter $=0.01(4)$.

\section{Acknowledgements}

We thank Dr. Xinghua Ma for assistance with the preparation of certain precursors to compound 6, the Australian Research Council for provision of a Scholarship to P.C.S., and the Institute of Advanced Studies for additional financial support.

[1] For reviews and useful points of entry into the relevant literature, see: a) S. F. Dyke, S. N. Quessy in The Alkaloids, Vol. 18 (Ed.: R. G. A. Rodrigo), Academic Press, New York, 1981, pp. 1-98; b) A. S. Chawla, V. K. Kapoor in The Alkaloids: Chemical and Biological Perspectives, Vol. 9 (Ed.: S. W. Pelletier), Elsevier Science Ltd., Oxford, 1995, pp. 86-153; c) Y. Tsuda, T. Sano in The Alkaloids, Vol. 48 (Ed.: G. A. Cordell), Academic Press, San Diego, 1996, pp. 249-337; d) H. Tanaka, T. Tanaka, H. Etoh, S. Goto, Y. Terada, Heterocycles 1999, 51, 2759.

[2] R. García-Mateos, M. Soto-Hernandez, D. Kelly, Biochem. Syst. Ecol. 1998, 26, 545.

[3] For a comprehensive listing of synthetic studies reported up until early 2003, see: a) H. I. Lee, M. P. Cassidy, P. Rashatasakhon, A. Padwa, Org. Lett. 2003, 5, 5067; for studies reported between 2003 and early 2006, see: b) P. C. Stanislawski, A. C. Willis, M. G. Banwell, Org. Lett. 2006, 8, 2143; for very recent studies, see: c) S. Gao, Y. Q. Tu, X. Hu, S. Wang, R. Hua, Y. Jiang, Y. Zhao, X. Fan, S. Zhang, Org. Lett. 2006, 8, 2373; d) I. Osante, M. N. Abdullah, S. Arrasate, N. Sotomayor, E. Lete, ARKIVOC 2007, $i v, 206$.

[4] a) M. G. Banwell, R. W. Gable, S. C. Peters, J. R. Phyland, J. Chem. Soc. Chem. Commun. 1995, 1395; b) M. Banwell, A. Edwards, J. Harvey, D. Hockless, A. Willis, J. Chem. Soc. Perkin Trans. 12000 , 2175 ; c) M. G. Banwell, J. E. Harvey, D. C. R. Hockless, A. W. Wu, J. Org. Chem. 2000, 65, 4241; d) M. G. Banwell, W. Ebenbeck, A. J. Edwards, J. Chem. Soc. Perkin Trans. 1 2001, 114; e) M. G. Banwell, J. E. Harvey, K. A. Jolliffe, J. Chem. Soc. Perkin Trans. 1 2001, 2002; f) M. G. Banwell, A. J. Edwards, K. A. Jolliffe, J. A. Smith, E. Hamel, P. Verdier-Pinard, Org. Biomol. Chem. 2003, 1, 296; g) R. M. Taylor, Aust. J. Chem. 2003, 56, 631; h) M. G. Banwell, M. O Sydnes, Aust. J. Chem. 2004, 57, 537; i) see reference [3b]; j) J. S. Foot, A. T. Phillis, P. P. Sharp, A. C. Willis, M. G. Banwell, Tetrahedron Lett. 2006, 47, 6817; k) M. G. Banwell, F. Vogt, A. W. Wu, Aust. J. Chem. 2006, 59, 415; 1) M. G. Banwell, A. T. Phillis, A. C. Willis, Org. Lett. 2006, 8, 5341; for a review of certain aspects of our work in this area, see: m) M. G. Banwell, D. A. S. Beck, P. C. Stanislawski, M. O. Sydnes, R. M. Taylor, Curr. Org. Chem. 2005, 9, 1589.

[5] N. Miyaura, A. Suzuki, Chem. Rev. 1995, 95, 2457.

[6] M. Banwell, D. Hockless, B. Jarrott, B. Kelly, A. Knill, R. Longmore, G. Simpson, J. Chem. Soc. Perkin Trans. 1 2000, 3555.

[7] a) L. A. Paquette, Z. Gao, Z. Ni, G. F. Smith, J. Am. Chem. Soc. 1998, 120, 2543; b) L. A. Paquette, M. J. Earle, G. F. Smith, Org. Synth. 1996, 73, 36. 
[8] a) M. Makosza, M. Wawrzyniewicz, Tetrahedron Lett. 1969, 10, 4659; for a discussion of the methods available for the generation of dihalocarbenes, see: b) M. G. Banwell, M. E. Reum in Advances in Strain in Organic Chemistry, Vol. 1 (Ed.: B. Halton), JAI Press, London, 1991, p. 19.

[9] L. Xu, U. H. Brinker in Synthetic Organic Sonochemistry (Ed.: J.-L. Luche), Plenum Press, New York, 1998, p. 344.

[10] R. K. Crossland, K. L. Servis, J. Org. Chem. 1970, 35, 3195.

[11] H. Kunz, C. Unverzagt, Angew. Chem. 1984, 96, 426; Angew. Chem. Int. Ed. Engl. 1984, 23, 436.

[12] K. Folkers, F. Koniuszy, J. Am. Chem. Soc. 1939, 61, 1232.

[13] R. B. Boar, D. A. Widdowson, J. Chem. Soc. B 1970, 1591.
[14] a) M. E. Jung, J. A. Berliner, D. Angst, D. Yue, L. Koroniak, A. D. Watson, R. Li, Org. Lett. 2005, 7, 3933; b) D. R. Deardorff, C. Q. Windham, C. L. Craney, Org. Synth. 1996, 73, 25.

[15] Z. Otwinowski, W. Minor in Methods in Enzymology, Vol. 276: Macromolecular Crystallography, Part A (Eds.: C. W. Carter, Jr., R. M. Sweet), Academic Press, New York, 1997, pp. 307-326.

[16] A. Altomare, G. Cascarano, C. Giacovazzo, A. Guagliardi, M. C. Burla, G. Polidori, M. Camalli, J. Appl. Crystallogr. 1994, 27, 435.

[17] P. W. Betteridge, J. R. Carruthers, R. I. Cooper, K. Prout, D. J. Watkin, J. Appl. Crystallogr. 2003, 36, 1487.

Received: May 2, 2007 Published online: August 10, 2007 